\title{
Statistical Methods for Detection and Mitigation of the Effect of Different Types of Cyber-Attacks and Inconsistencies in Electrical Design Parameters in a Real World Distribution System
}

Vivek Joshi

Follow this and additional works at: https://researchrepository.wvu.edu/etd

\section{Recommended Citation}

Joshi, Vivek, "Statistical Methods for Detection and Mitigation of the Effect of Different Types of CyberAttacks and Inconsistencies in Electrical Design Parameters in a Real World Distribution System" (2014). Graduate Theses, Dissertations, and Problem Reports. 7099.

https://researchrepository.wvu.edu/etd/7099

This Thesis is protected by copyright and/or related rights. It has been brought to you by the The Research Repository @ WVU with permission from the rights-holder(s). You are free to use this Thesis in any way that is permitted by the copyright and related rights legislation that applies to your use. For other uses you must obtain permission from the rights-holder(s) directly, unless additional rights are indicated by a Creative Commons license in the record and/ or on the work itself. This Thesis has been accepted for inclusion in WVU Graduate Theses, Dissertations, and Problem Reports collection by an authorized administrator of The Research Repository @ WVU. For more information, please contact researchrepository@mail.wvu.edu. 


\title{
Statistical Methods for Detection and Mitigation of the Effect of Different Types of Cyber-Attacks and Inconsistencies in Electrical Design Parameters in a Real World Distribution System
}

\author{
By \\ Vivek Joshi \\ Thesis submitted to the \\ Benjamin M. Statler College of Engineering and Mineral Resources \\ at West Virginia University \\ in partial fulfillment of the requirements for the degree of \\ Master of Science \\ in \\ Electrical Engineering \\ Dr. Jignesh Solanki, Ph.D., Chair \\ Dr. Sarika Khushalani Solanki, Ph.D. \\ Dr. Radhey Sharma, Ph.D. \\ Lane Department of Computer Science and Electrical Engineering \\ Morgantown, West Virginia \\ 2014
}

Keywords: Distribution System, Capacitor Control, DG, MLR, System modelling parameter inconsistencies, Deception Attack, Load Redistribution Attack, OpenDSS.

Copyright 2014 Vivek Joshi 


\title{
ABSTRACT \\ Statistical Methods for Detection and Mitigation of the Effect of Different Types of Cyber- Attacks and Inconsistencies in Electrical Design Parameters in a Real World Distribution System
}

\author{
Vivek Joshi \\ Master of Science in Electrical Engineering \\ West Virginia University \\ Advisor: Dr. Jignesh Solanki, Ph.D.
}

In the present grid real time control systems are the energy management systems and distribution management systems that utilize measurements from real-time units (RTUs) and Supervisory Control and Data Acquisition (SCADA). The SCADA systems are designed to operate on isolated, private networks without even basic security features which are now being migrated to modern IP-based communications providing near real time information from measuring and controlling units. To function "brain" (SCADA) properly "heart" (RTUs) should provide necessary response thereby creating a coupling which makes SCADA systems as targets for cyber-attacks to cripple either part of the electric transmission grid or fully shut down (create blackout) the grid. Cyber-security research for a distribution grid is a topic yet to be addressed. To date firewalls and classic signature-based intrusion detection systems have provided access control and awareness of suspicious network traffic but typically have not offered any real-time detection and defense solutions for electric distribution grids.

This thesis work not only addresses the cyber security modeling, detection and prevention but also addresses model inconsistencies for effectively utilizing and controlling distribution management systems. Inconsistencies in the electrical design parameters of the distribution network or cyber-attack conditions may result in failing of the automated operations or distribution state estimation process which might lead the system to a catastrophic condition or give erroneous solutions for the probable problems. This research work also develops a robust and reliable voltage controller based on Multiple Linear Regression (MLR) to maintain the voltage profile in a smart distribution system under cyber-attacks and model inconsistencies. The developed cyber-attack detection and mitigation algorithms have been tested on IEEE 13 node and 600+ node real American electric distribution systems modeled in Electric Power Research Institute's (EPRI) OpenDSS software. 


\section{ACKNOWLEDGEMENTS}

I would like to express my sincere gratitude to my advisor, Dr. Jignesh Solanki, for his invaluable guidance, support, and encouragement. I thank Dr. Jignesh for believing in me that I can accomplish this goal with hard work and sincere effort. I thank him for providing me that confidence which lead to completion of this research work. Next, I would like to thank my second committee member Dr. Sarika Khushalani Solanki. It was from her that I learned the difference between mere hard work and hard work with focus and dedication. Both Dr. Sarika Khushalani Solanki and Dr. Jignesh Solanki have been with me, guiding me throughout my two years of research. I would also like to thank my other committee member Dr. Radhey Sharma, whose feedback and reviews helped me improve the quality of this thesis.

I would like to thank my parents for their moral support throughout my graduate studies. Their love and affection have helped me overcome the toughest of challenges during these two hard years. Lastly, but in no sense the least, I am thankful to all my friends who made my stay at the West Virginia University a memorable and valuable experience. 


\section{Contents}

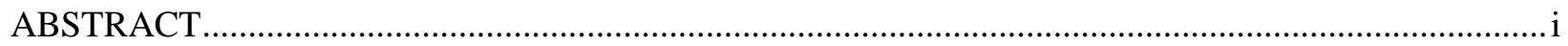

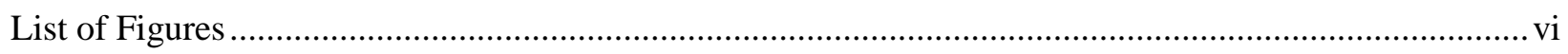

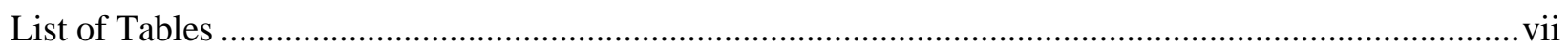

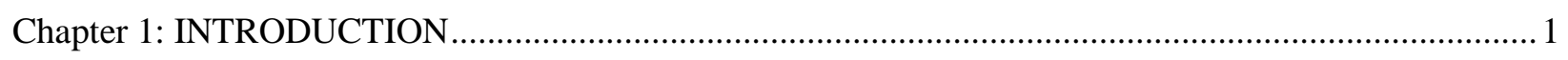

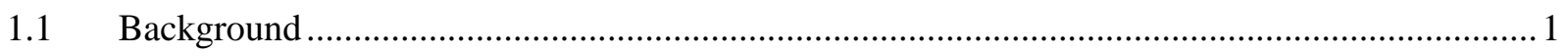

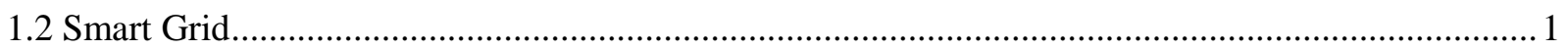

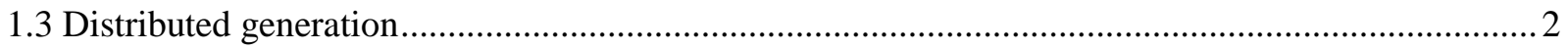

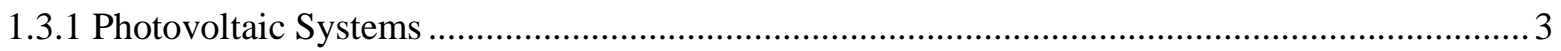

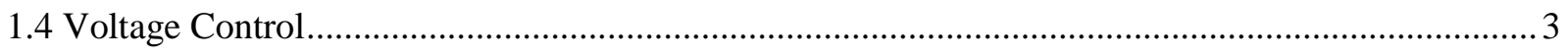

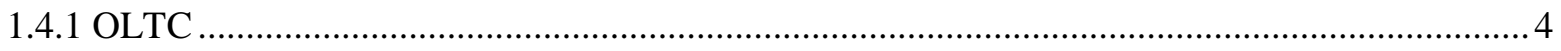

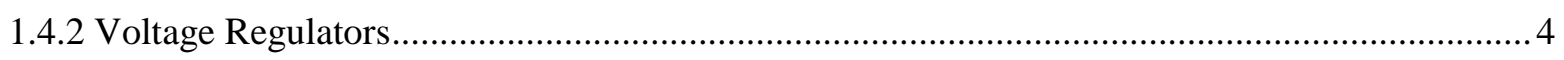

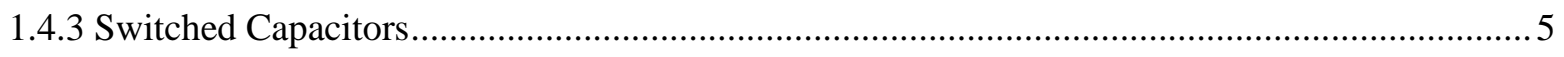

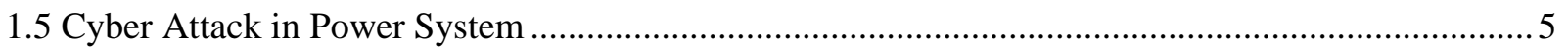

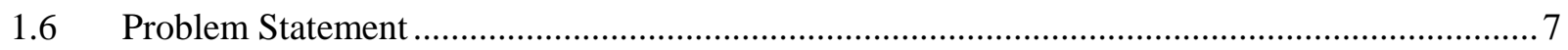

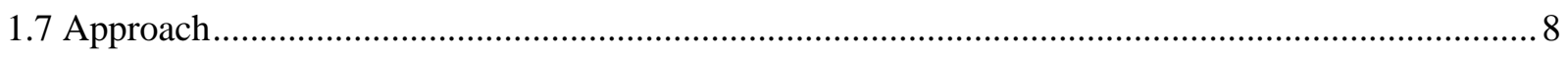

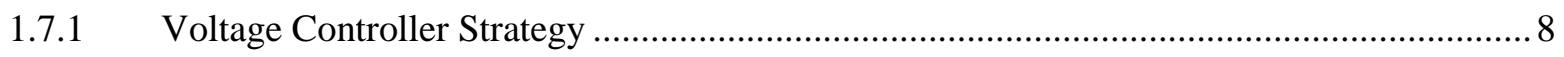

1.7.2 Cyber Attack Detection Algorithm …................................................................... 8

1.7.3 Electrical Design Parameters Inconsistency effect on Losses Calculation ......................... 9

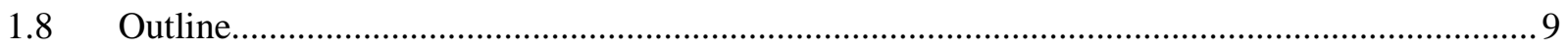

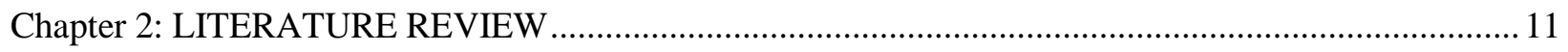

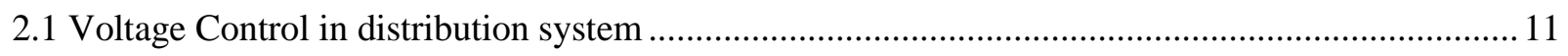

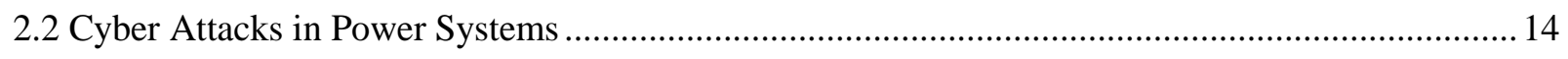

Chapter 3: MATHEMATICAL MODEL AND FORMULATION ...................................................... 19

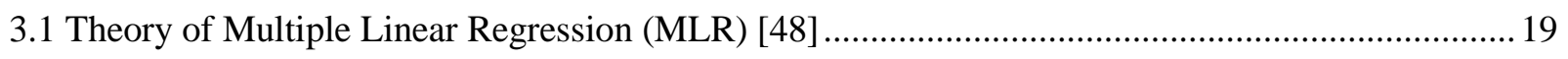

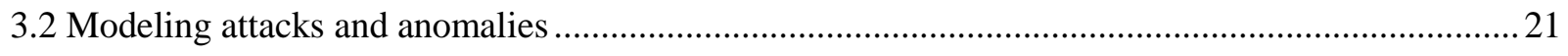

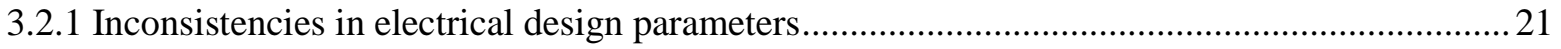




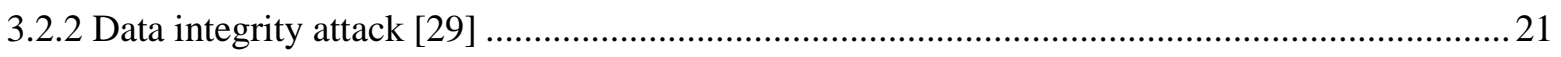

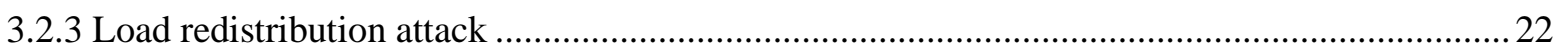

3.3 Distributed Cyber Attack Detection Method ...............................................................................22

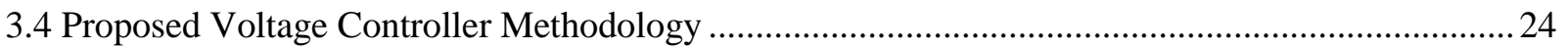

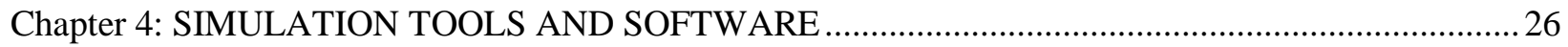

4.1 Open Distribution System Simulator (OpenDSS) ...........................................................26

4.1.1 Extensive Range of Solution Modes ....................................................................... 28

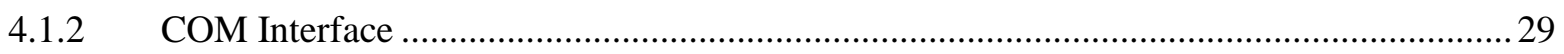

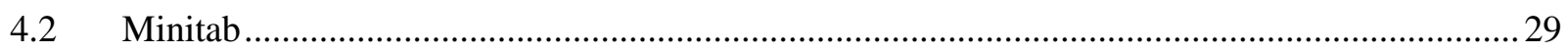

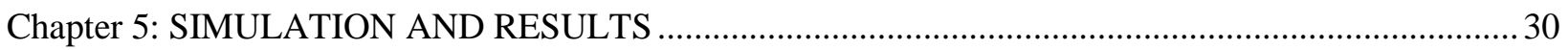

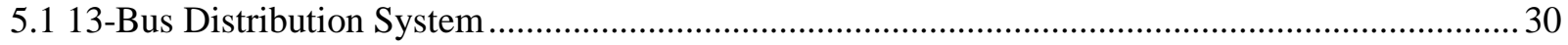

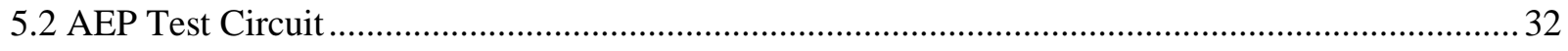

5.2.1 MLR based Statistical Voltage Controller ...................................................................... 33

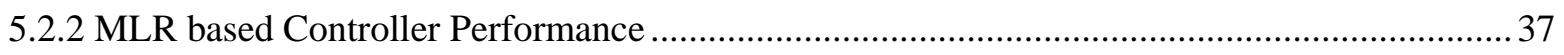

5.2.3 Cyber Attack Distributed Detection Method ..................................................................... 40

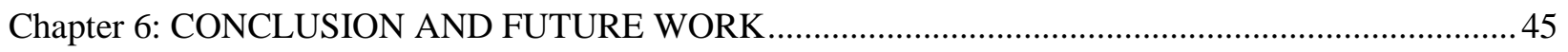

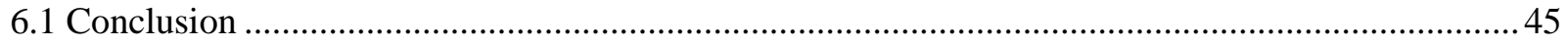

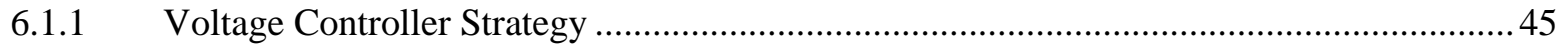

6.1.2 Distributed cyber-attack detection technique ........................................................... 45

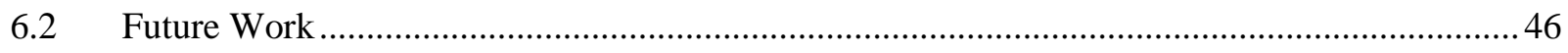

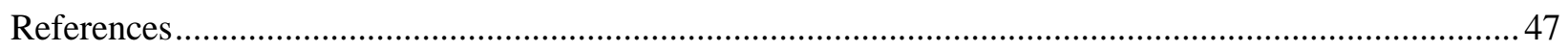




\section{List of Figures}

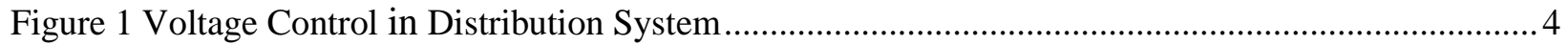

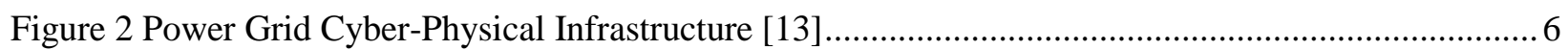

Figure 3 Divided Areas based on Reactive Power Domains [24] ........................................................ 13

Figure 4 Cyber-Attack on Control System [29] .............................................................................. 15

Figure 5 Deception Attack on State Estimator in a Power Grid [34].................................................... 16

Figure 6 SCADA Controlled Voltage Loop in a Transmission System [44] ............................................. 17

Figure 7 Detection of Cyber-Attack with Local Agents for each Area [40]............................................ 18

Figure 8 A Statistical Reactive Power Model Algorithm ....................................................................25

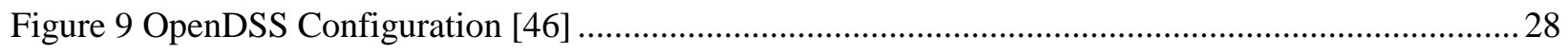

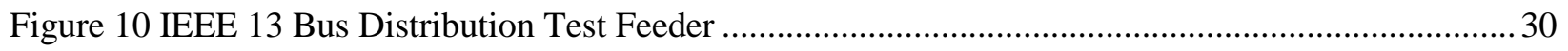

Figure 11 Normal Probability Plots for the Regression Models for Buses 652 and 684 .........................31

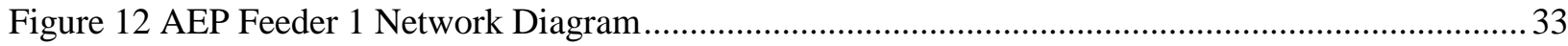

Figure 13 Normal Probability Plots for the Regression Models for Buses 164_west, 146_west, 143_west

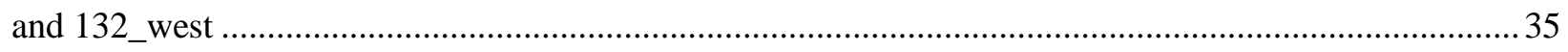

Figure 14 Controller Validations for the Kvar Calculation Models for the Buses 164_west, 146_west,

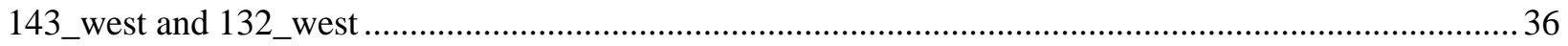

Figure 15 Losses in Four Maximum Losses giving Lines with Each Type of Line Geometry for 2-

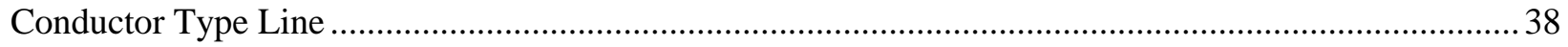

Figure 16 Losses in Four Maximum Losses giving Lines with Each Type of Line Geometry for 4-

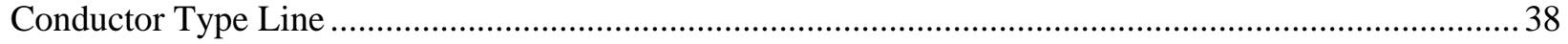

Figure 17 Controller Testing for the Voltage Controllers against Inconsistencies in Electrical Design

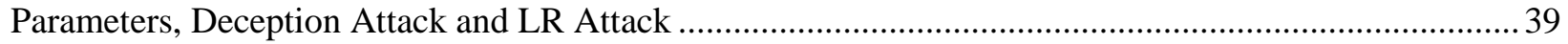

Figure 18 Cluster Division for AEP Feeder 1 Network ...................................................................... 41

Figure 19 Normal Probability Plots for the Regression Models of the Local Agents ............................. 42

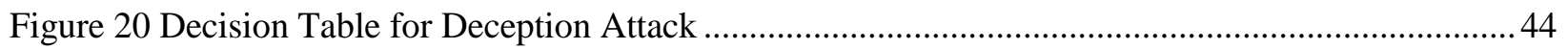

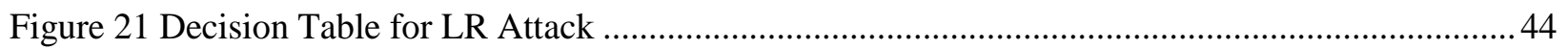




\section{List of Tables}

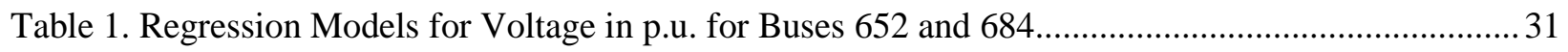

Table 2. Controller Validation for the kVar Calculation Models ............................................................ 32

Table 3. Regression Models for Voltage in p.u. for Buses 164_west, 146_west, 143_west and 132_west34

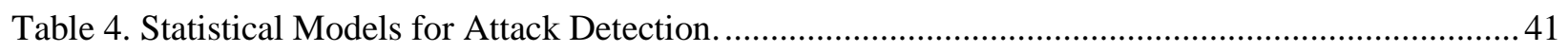

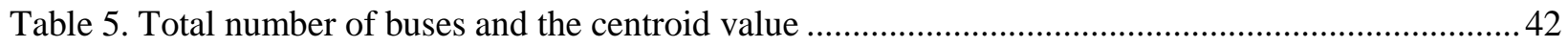




\section{Chapter 1: INTRODUCTION}

\subsection{Background}

The electric power system consists of three fields- generation, transmission and distribution; which are constantly evolving to supply the ever increasing demand in a more cost effective, efficient and reliable manner, both for the utilities and the customers. For this purpose the electric power grid has become the most complex and highly invested industry undergoing constant technological renovations. These technological advancements led to the concepts of SCADA, Energy Management systems (EMS), Distribution System Management (DMS), Smart Grid and Distribution Automation (DA) in the power grid.

\subsection{Smart Grid}

The concept of a smart grid started with the formation of Independent System Operator (ISO) and Regional Transmission Organization (RTO) under the recommendation of Federal Energy Regulatory Commission (FERC). The ISOs and RTOs are formed to make a smarter electrical grid keeping in mind the demands of the $21^{\text {st }}$ century. The US Department of Energy (DOE) defines the overall vision of Smart Grid as the following [1].

1. Intelligent Automation- having sensors to sense overload conditions and rerouting power and avoiding outage conditions; automatic isolation of faulted areas with minimum disruption of power.

2. Smooth Integration of Distributed Generation (DG) - integration of any fuel source including solar and wind as easily and transparently as coal and natural gas; also other technologies like energy storage.

3. Sophisticated Demand Response Capabilities - supporting real-time communication between the consumer and utility so consumers can alter their energy consumption based on individual inclinations, like price and/or environmental concerns. 
4. Quality-centric - capable of delivering the power which is free of sags, spikes, disturbances and interruptions.

5. Robust - highly resistant to cyber-attack and natural disasters as it becomes more decentralized.

There are vast benefits to the country with the commencement of Smart Grid [2]. The chances of cascading outages and dependency on foreign fuel are reduced. One of the important objectives of smart grid concept is to allow high penetration of DG and new storage technologies into the present grid smoothly. DGs are small scale power generation technologies located close to the load having capabilities of lowering costs, improving reliability and reducing emissions.

\subsection{Distributed generation}

With the advent of smart grid and advancement of new technologies, the utilities are focused towards adding DG into their existing infrastructure. The addition of DG does bring along different technological and environmental benefits to the power grid like locally fulfilling the consumer demands, reducing power losses and avoiding transmission and distribution system expansion [3]. Earlier conventional power sources were used for these purposes but in the last few years, renewable energy has taken their place as a feasible future source of electric energy as they can eradicate the problems of increasing consumer demand, fluctuating fossil fuel prices and also solve problems related to environmental issues. The prevalent forms of DG are wind power, solar photovoltaic, fuel cells and micro-turbines. The DGs that are of electromechanical type could be directly interfaced whereas other DGs require inverter based systems to connect to the power grid.

Although there are many advantages of integrating DGs into the grid there are some negative impacts too. The integration of DGs changes the unidirectional power flow of a traditional radial distribution network to a two-way power flow because of the addition of generators in the distribution side [4]. This also affects the traditional relays and protection devices as they generally do not have directional capabilities. The power quality can also be affected as DG

devices are connected to the power grid by power electronic devices which might cause distortion of the current and voltage waveforms and induce harmonics [5]. 


\subsubsection{Photovoltaic Systems}

Solar energy is the world's most copiously available form of renewable energy source and so photovoltaic generators are one of the fastest emerging DG technologies, with an estimate annual growth rate of $25-35 \%$ in the power market [1]. The reason for this remarkable growth in spite of their high installation cost can be given to the advancements in power electronics field, storage devices, etc. which are highly essential for large scale installation of PV generators at distribution side. Moreover PV generators are vigorously being endorsed in order to alleviate environmental issues such as the greenhouse effect and air pollution and also they help in relieving thermal overloads and reducing losses in distribution systems [6-7]. Another advantage is that solar technology is very flexible and can be easily changed to provide the required power for different loads. The energy produced by the PV system reduces the apparent load whereas the surplus energy flows into the grid. But one drawback that these solar generators cause when they installed near to the load side owing to their intermittent nature even after so much effort has been done to get an accurate estimate of the daily solar generation curve is that they disturb the voltage profile of the distribution system.

\subsection{Voltage Control}

The simplest voltage control methods in a distribution system use local measurements for maintaining the voltage profile and data transfer between the distribution nodes is not required. On the contrary, there are methods that require data transfer between the distribution nodes and they determine their control actions based on the information of the entire distribution network. These methods are known as coordinated voltage control methods. A variety of coordinated or centralized voltage controls methods have been developed in distribution systems with different levels of effectiveness, complexity, communications requirements, and cost effectiveness. Centralized Distribution Management System (DMS) control and also coordination of distribution network components such as OLTC, voltage regulators, DGs and switched capacitor control are some of the examples of coordinated voltage management for distribution systems. Figure 1 show the different elements employed in a distribution system for the purpose of controlling the voltage. 


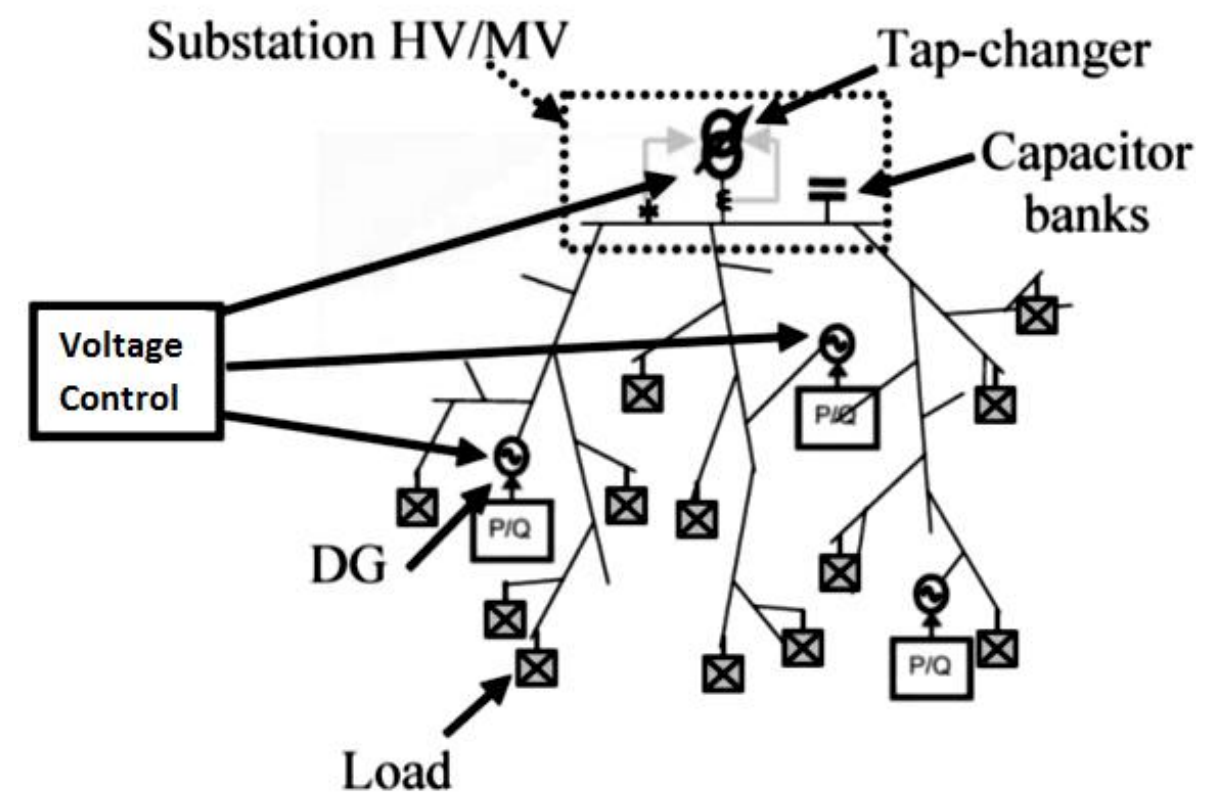

Figure 1 Voltage Control in Distribution System

\subsubsection{OLTC}

A tap changer is a device for regulation of the output voltage to required levels fitted to power transformers. This is typically achieved by altering the ratios of the transformers on the system by varying the number of turns in one winding of the suitable transformer/s. Tap changers offer flexible control to keep the voltage supply within the limits. Tap changers can be either on load or off load. On load tap changers transfer current from one voltage tap to the next without interrupting the supply. Tap changers can be adjusted to fit the application requirements.

\subsubsection{Voltage Regulators}

A voltage regulator is a device used to automatically maintain a constant voltage level in electric power distribution system. It may be installed at a substation or along distribution lines so that all customers receive steady voltage without worrying about how much power is drawn from the line. Generally voltage regulators have capability of raising or lowering the voltage by $10 \%$. 


\subsubsection{Switched Capacitors}

The main use of shunt capacitor banks (SCB) is to provide capacitive reactive compensation or power factor correction. The reason for increased use of SCBs is their inexpensiveness, easy and quick installation and easy deployment almost anywhere in the network. Other valuable effects of its installation on the distribution system are: improvement of the voltage at the load, improved voltage regulation, reduction of losses and cost savings due to delay of investments in transmission system.

\subsection{Cyber Attack in Power System}

The numerous technological advancements and increasing demand for reliable energy have stimulated the development of a smart grid. The smart grid is supposed to expand the present capabilities of the power grid's generation, transmission and distribution systems to support the requirements of distributed generation, renewable energy resources, electric vehicles and demand-side management of power.

The present distribution system will see the advent of advanced technologies such as phasor measurement units (PMU), wide area measurement systems, substation automation, and advanced metering infrastructures (AMI) to realize these objectives. But their introduction to the distribution side also presents an increased reliance on cyber resources which may be susceptible to attack conditions. Moreover, power grid in the past decade has encountered numerous cyber related attacks which have elevated the interrogation regarding the security susceptibilities and its large scale impact on the critical power grid infrastructure [8-12]. The cyber-physical infrastructure of a power grid in shown in figure 2. 


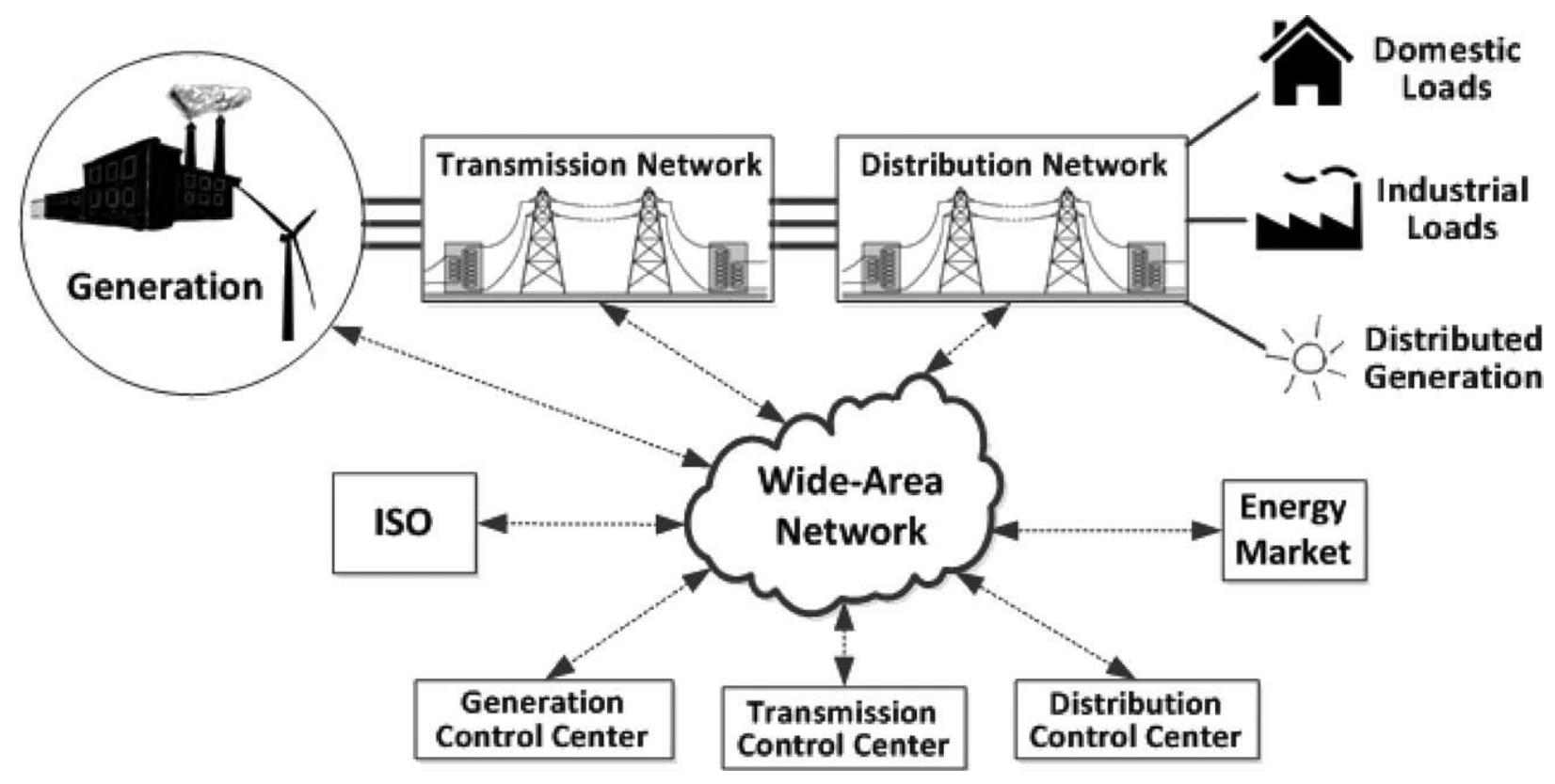

Figure 2 Power Grid Cyber-Physical Infrastructure [13]

The different types of attacks that can affect the normal operation of a power distribution system as described in [14] are:-

1) Denial of Cooperative Operation (DoS): In this attack, the communication channels are jammed by flooding them with junk packets which can result in loss of important data and might affect the automated control operations.

2) Desynchronization attacks: In this attack, the control algorithms of automated operation which are time dependent are attacked.

3) Data Injection Attacks: In this attack, false operational data such as status or control information are send that can significantly affect the operations of a power grid. This type of attack requires thorough knowledge of the communication protocol. 


\subsection{Problem Statement}

The primary objective of this thesis is to develop a robust and reliable voltage controller based on multiple linear regressions to maintain the voltage profile in a distribution system with distributed generators (DG) connected to it. Regression is based on least squares method making use of data acquired from exact simulations of the distribution network. The independent variables selected for this study are active power output of DG, total load and var injection at the capacitor bank as independent variables whereas the dependent variable is per unit voltage at the violating buses. The proposed controller is validated on IEEE 13 bus distribution system and American Electric Power System feeder modeled in OpenDSS.

This thesis also models two different types of attack, namely data integrity attack on the voltage control loop and load redistribution attack and also inconsistencies in electrical design parameter in a power distribution network. A regression based distributed detection algorithm having local detection agents is developed for detection of cyber-attack in a distribution system with DG connected to it. An algorithm is proposed to select a certain number of buses in the system to be declared as elected buses and linear regression based local agents are developed from the elected buses. The cyber-attacks and detection technique are developed in AEP feeder modeled in OpenDSS.

This thesis also validates the effectiveness of the proposed voltage controller strategy against data integrity attack on voltage control loop, load redistribution attack and inconsistencies in electrical design parameter in a power distribution network which are modeled in AEP Distribution feeder.

The effect of inconsistency in electrical design parameter in calculation of the line losses for overhead lines in AEP Feeder without any DG is presented. The parameter considered for modeling this inconsistency is line reactance which is modeled in OpenDSS.

A few assumptions made in this thesis work are listed below,

- Voltage regulators are turned off in both IEEE 13 bus distribution system and AEP feeder for all the proposed strategies. 
- Data sets for the independent variables and dependent variables for the regression are taken from the exact simulation of the distribution system in OpenDSS.

\subsection{Approach}

The sections to follow will develop voltage controller model, cyber-attacks and distributed cyber- attack detection technique. The key aspects of this research are highlighted in subsections below.

\subsubsection{Voltage Controller Strategy}

Integration of DG to a distribution system and the changing load conditions impact voltage profile in a distribution system. So their affect should be taken into account while developing a strategy for voltage regulation in a distribution system.

- $\quad$ Exact models of the distribution systems are modeled in OpenDSS for the analysis.

- Multiple linear regression is done in Minitab with per unit voltage at violating buses as dependent variables and active power output of DG, total load and var injection form the capacitor bank as independent variables.

- Data sets for the dependent and independent variables are generated from OpenDSS using random network and loading conditions.

- Optimum var settings for capacitor banks are calculated in Matlab using the regression models of per unit voltage of violating buses.

\subsubsection{Cyber Attack Detection Algorithm}

An agent based detection technique for cyber-attacks is developed based on linear regression. This technique can be effectively used until the topology of the distribution system changes and can detect different types of attacks, namely data integrity attack on voltage control loop and load redistribution attack.

- $\quad$ Exact models of the distribution systems are modeled in OpenDSS for the analysis.

- $\quad$ Cyber-attacks are modeled in OpenDSS in the exact model itself. 
- A distributed algorithm using local agents based on linear regression is developed for the detection of the cyber-attacks. The linear regression analyses in done in Minitab software.

- The detection technique is tested for different cases of cyber-attacks in AEP feeder.

\subsubsection{Electrical Design Parameters Inconsistency effect on Losses Calculation}

The effect of inconsistency in electrical design parameter in calculation of the line losses for overhead lines in AEP Feeder without any DG is presented. The parameter considered for modeling this inconsistency is line reactance which is modeled in OpenDSS.

- The AEP feeder 1 is studied thoroughly and all the different conductor type lines are identified.

- $\quad$ The different types of geometries used for all the different types of lines are identified in the AEP feeder 1.

- Losses for a few maximum losses giving lines of a 2 conductor type line and 4 conductor type line are calculated with all the identified geometries for those types of lines including their original geometry.

- The effect of different types of geometries on different types of lines is analyzed.

\subsection{Outline}

The outline of the remaining chapters is given in this section.

Chapter 2 is a comprehensive literature review on voltage control in distribution system using voltage regulators, OLTC, capacitor banks, DGs or a combination of any of these. A literature review on the different cyber-attacks in power system is also discussed in details.

Chapter 3 is about mathematical formulation of multiple linear regression technique, modeling of the inconsistencies in the electric design parameters, data integrity attack on voltage control loop and load redistribution attack in a distribution system. The voltage controller strategy and cyberattack detection technique is also formulated.

Chapter 4 gives a comprehensive description of the software packages used in this study. The advantages presented by the software to the pertinent applications are also discussed. 
Chapter 5 presents the test systems, simulations for voltage control strategy and associated regression models and the cyber-attack detection technique and the associated regression models. The effect of the cyber-attacks on voltage control in a distribution system is also presented.

Finally, chapter 6 lists conclusion of this study and provides scope for future work. 


\section{Chapter 2: LITERATURE REVIEW}

This chapter reviews the literature for voltage control using capacitor banks, OLTC, voltage regulators and DG. Also deception attack and load distribution attack in power systems.

\subsection{Voltage Control in distribution system}

Different strategies exist in the present distribution system which can be used by DMS or DA to counter the voltage instability like regulator control using different tap settings, capacitor control supplying/ absorbing Kvar to/from the distribution system, etc. Capacitor control is usually done to achieve the following goals: reduce losses due to reactive load current, reduce kVA demand, decrease customer energy consumption, improve voltage profile, and increase revenue.

A lot of research has been done on the use of capacitor control, both in offline and online mode to maintain the voltage profile in a distribution system. Reference [15] discussed about the traditional capacitor bank control strategies which mainly employed timers, voltage controls, and voltage with time bias; their advantages and drawbacks. Also some improved capacitor control technologies like Fisher Pierce Current Control, Beckwith Electric Co. Inc., RTE Combinational Capacitor Control and lastly proposed an exhaustive search methodology for determining the optimum set points for the capacitor bank switching.

A control strategy for the control of both voltage and reactive power at distribution substation and feeder level using an optimization method involving minimizing the sum of weighted squares of the set points values for all the variables that need to be controlled is proposed in [16]. The variables chosen to be controlled for this study are voltage, reactive power and feeder losses.

Reference [17] provides the optimum placement, replacement and control of capacitors in distribution systems using a two stage algorithm; one stage being Genetic Algorithm to find neighbourhoods of high quality solutions and the second stage is for improving the solution given by first stage. Reference [19] proposes solutions for the problems of optimal location, type, and size of the capacitors in a radial distribution system taking voltage constraints and load variations in the optimization problem. The problem is formulated as a mixed integer linear programming problem. 
Reference [22] presents the problem formulation, solution methodology and mathematical validation of a novel capacitor placement and their real time control schemes in an unbalanced distribution system. The problem is divided into two subproblems, first the capacitor placement and second the real time control. The approach of quadratic integer programming is used which yielded the optimum number, location and size of capacitor to be installed in the system. The numerical studies for this are presented in [23].

The maintenance of voltage profile at every customer's meter within the ANSI standards using step up voltage regulators is shown in [18]. The regulators are considered as tap-changing autotransformers. Reference [20] provides control of the local regulating devices in a distribution substations and feeders taking dynamically changing system conditions into account instead of the local measurements.

The problem of minimizing the real power losses and enhancing the voltage profile in a distribution system is solved using a reconfiguration methodology built on a new adaptive imperialist competitive algorithm in [21].

Distributed capacitor control methodologies to regulate voltage profile in a distribution system are performed in [24-25] in which the whole network is divided into different control zones based on reactive power area of each governable shunt capacitor and finding the optimal setting point for each zone. The figure 3 shows how the areas are divided based on reactive power domains.

Reference [26] provides an optimal vol/var control strategy with solid state transformers (SST) for var compensation and voltage regulators for voltage regulation where the final objective is to minimize total system power loss while maintaining the voltage profile within the permissible limits in a distribution system. Distributed generation is taken into account while making an optimal capacitor control algorithm for getting the optimum set point in [27]. Different types of DGs are considered with their different mathematical models for this study. 


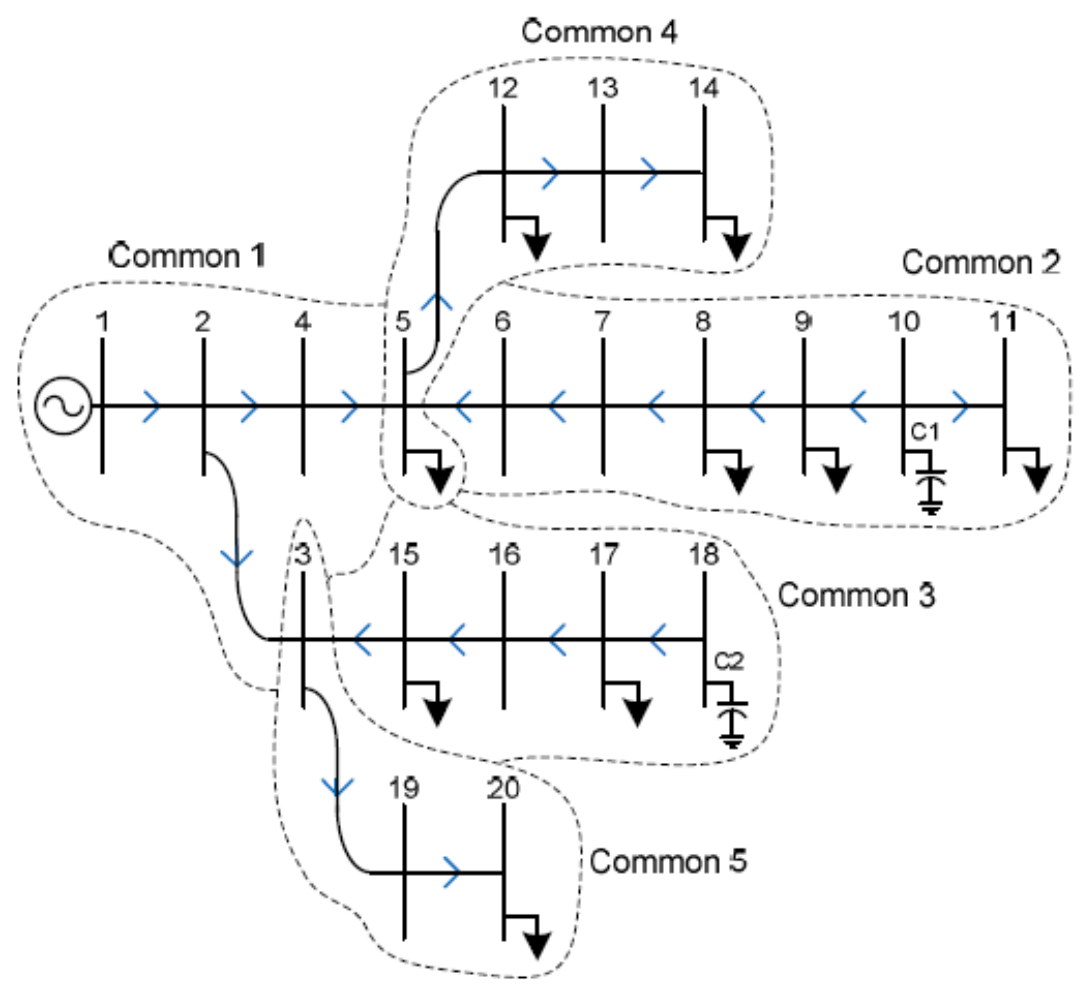

Figure 3 Divided Areas based on Reactive Power Domains [24]

Both offline and online modes have some disadvantages. In offline mode, the control switches the capacitor bank according to some predefined time which may or may not relate with actual loading conditions because it has no feedback mechanism to monitor system conditions, so at low loading conditions the vars may be supplied and vice versa. The online mode requires continuous monitoring of either the power factor of the total current supplied to the distribution system or some other quantity by some microprocessor-based relays due to the continuous change in loading conditions and intermittent DG penetration, if it is present, which can become expensive for getting really accurate measurements. Also, the computation becomes complex as the whole system has to be solved each time the var injections need to be calculated. Moreover, if there is any inconsistency present in the electrical design parameters of the distribution system while calculating the required var injections, or under cyber- attack conditions the capacitor control will become impractical. 


\subsection{Cyber Attacks in Power Systems}

With the rapidly increasing penetration of distributed generations (DG), the need for demand side management, and control of industrial and residential loads through demand response has led to the emergence of Distribution Management Systems (DMS) by electric utilities for better analysing and controlling the distribution systems. DMS rely heavily on the cutting edge communication technologies, advanced sensors, and other automated systems to achieve realtime adjustment to changing loads, generation, and fiasco conditions of the distribution system. Improving the reliability and quality of service, maintaining acceptable frequency and voltage levels in the distribution system usually without the operator intervention are the main functions of these systems.

However, these advanced systems also craft new vulnerabilities in power infrastructures. Different types of attacks have been researched in the past for the smart grid's automated control systems, wide area measurement and supervisory control and data acquisition systems. For instance, in references [28-29] deception attacks and denial of service (DOS) attacks against a networked control system are defined. Deception attacks refer to the possibility of compromising the integrity of control packets or measurements, and they are cast by altering the behavior of sensors and actuators. Figure 4 shows the various points at where a cyber-attack can be done in a control system where A1 and A3 are the data integrity attacks, A5 is an attack on the physical system and A2 and A4 are attacks on the communication links connecting the physical system with the controller. 


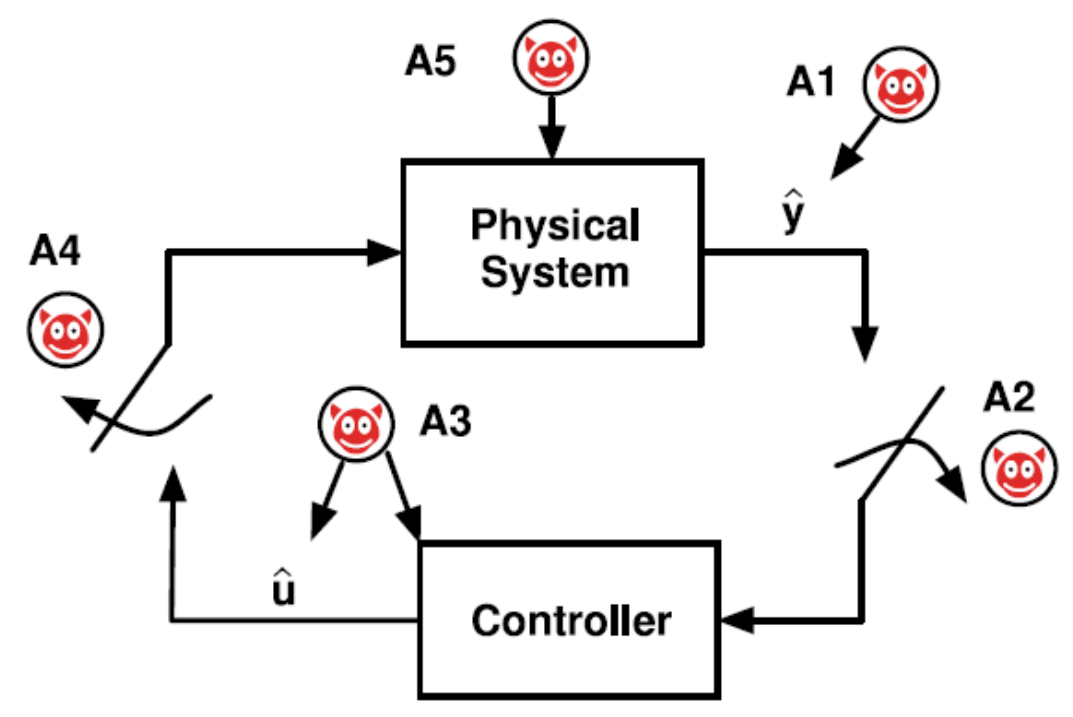

Figure 4 Cyber-Attack on Control System [29]

Similarly, specific deception attacks in the context of static estimators through SCADA systems, known as false data injection attacks (FDIA) are described in [30-34].In FDIA an adversary aims to hack the readings of multiple sensors to mislead smart grid's decision making process. Reference [30] shows modeling of a FDIA with incomplete knowledge of the power grid parameter and real time attributes such circuit breaker positions, tap positions of the voltage regulators, etc. It was proved that even if the attacker does not have access to full network information the attack can still bypass the bad data detection techniques of the state estimator. Also it has been shown in [32] that if the attacker knows the complete network conditions, the FDIA cannot be detected by the bad data detecting techniques used by the present state estimation systems. Reference [31] proposes a novel analytical method for doing the vulnerability analysis of state estimation when it is under FDIA on the SCADA system of an electric grid. Reference [34] also presents a deception attack on the state estimator through SCADA with a perturbed or outdated model of the electric grid. Both linear and non-linear state estimators are considered in this study. It was shown the more the accurate information the attacker possesses the greater the threat of a deception attack becomes. Figure 5 shows a schematic diagram of deception attack on state estimator in a power grid. 


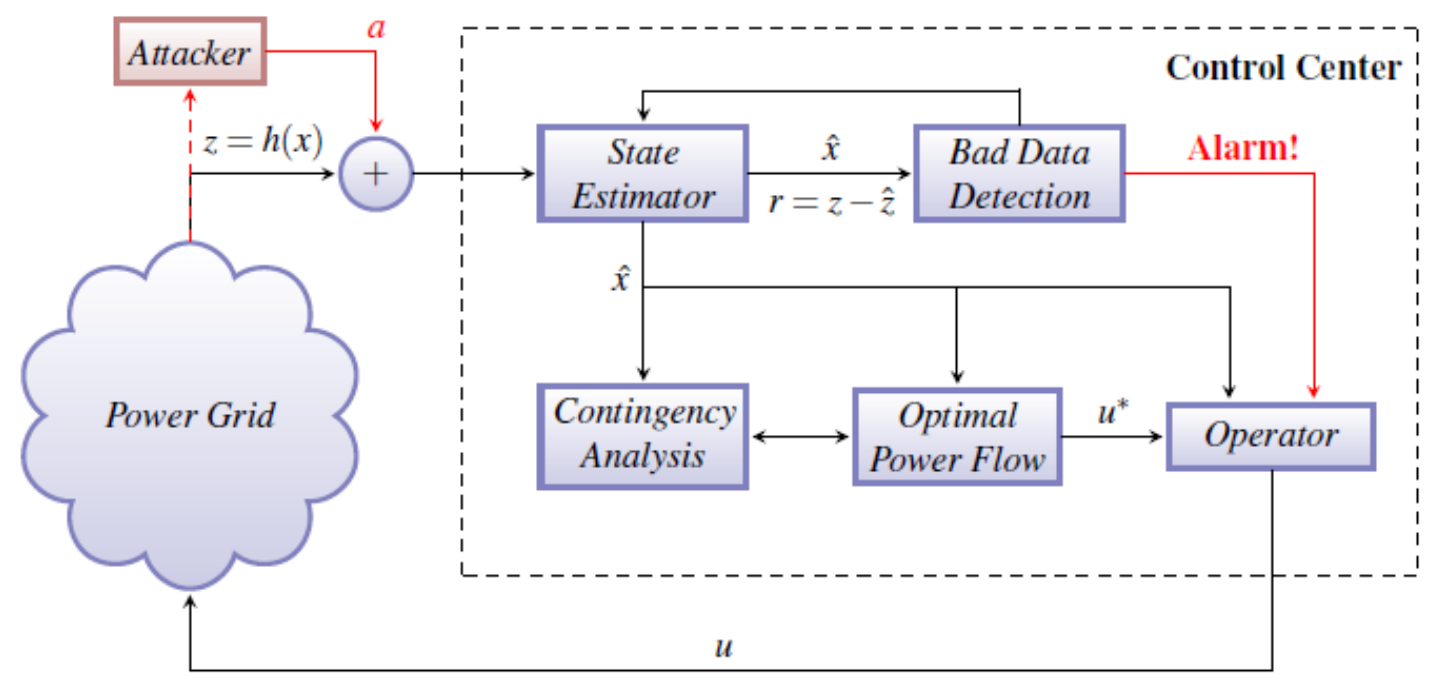

Figure 5 Deception Attack on State Estimator in a Power Grid [34]

Another subclass of the FDIA is load redistribution (LR) attack which has been recently studied in [35-37]. Reference [35] models a LR attack which is an attack on the smart meters of a smart grid changing the load at different buses in the system but maintaining the total load as it is. The effect of this attack on security constrained economic dispatch (SCED) has been discussed. Also it was proved that these LR attacks can bypass the bad detection schemes in if the network conditions are known.

Reference [36] shows that an LR attack can be modeled which can bypass the bad data detection techniques presently available even under incomplete network information. Quantitative analysis of the damage that LR attacks can do to the power system operations and security is discussed in [37] and the prevention measures are also provided.

The effects of data integrity cyber-attacks on the SCADA controlled voltage control loop of a transmission system is shown in [44]. The voltage control provided by FACT devices is targeted. The paper also explains by sensitivity analysis technique which device to attack to affect which bus. Figure 6 explains the schematic of a SCADA controlled voltage loop in a transmission system. Similarly the effect of cyber-attack on automatic generation control loop is presented in [43]. 


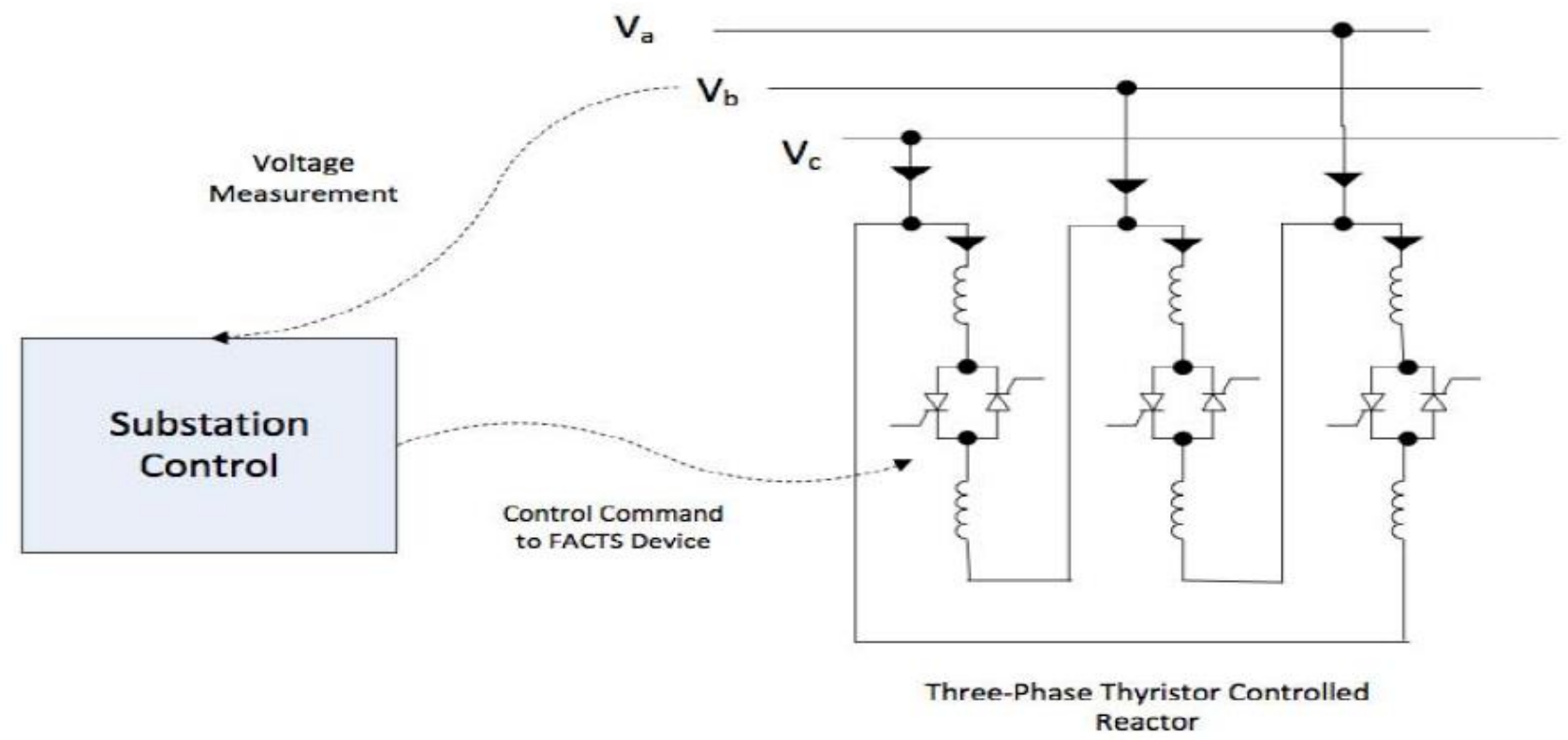

Figure 6 SCADA Controlled Voltage Loop in a Transmission System [44]

With numerous diverse imminent cyber threats to the automated control systems and state estimators of smart grids, the defensive mechanisms also have to build. Intuitively, there are two approaches to protecting control applications of power grid. The first is to design robust control algorithms that can detect or tolerate malicious data modification. The second is to protect the sensor measurements and other data from being manipulated.

A lot of literature is available on the detection of cyber-attacks on state estimators through SCADA systems [38-40]. Reference [38] proses a method of cyber-attack detection taking the information from both the active power measurements and the reactive power measurements. It was observed that this strategy pin pointed the exact transmission lines attacked. In [39] authors propose a strategy of protecting a selected set of sensor measurements for detecting the attack by verifying somehow those set of measurements. Reference [40] describes s fully distributed procedure for the detection of cyber-physical attacks in power networks. In this study, the network is divided into different areas as shown in figure 7 and then each area is monitored and controlled by a local control center. A detection filter is designed based on sparse residual filter in descriptor form. 


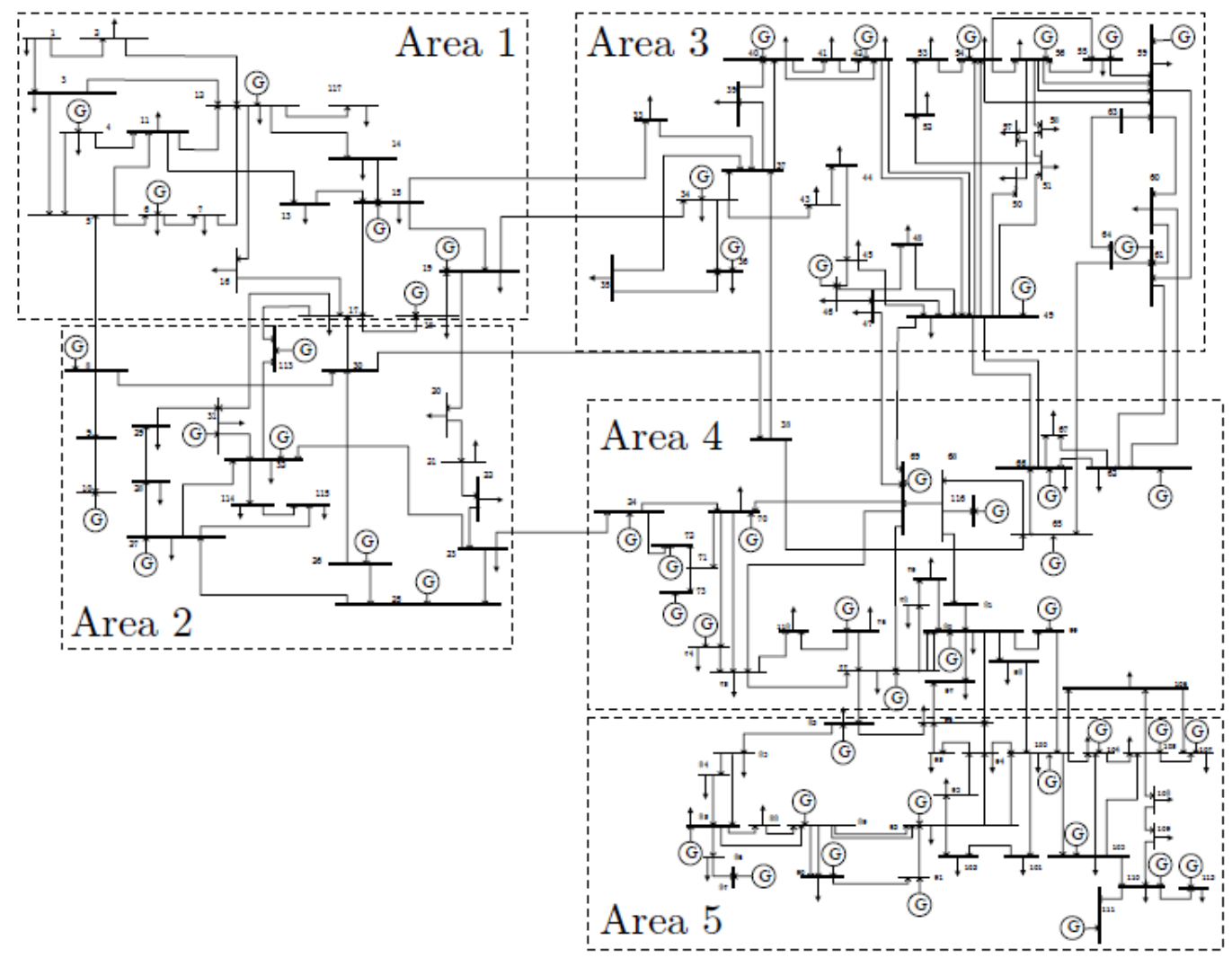

Figure 7 Detection of Cyber-Attack with Local Agents for each Area [40]

References [41-42] propose physically protecting a certain number of the PMU out of the total which is a tough task. Reference [41] using graph algorithms provides with the minimum number of PMUs that need to be physically protected in order neutralize a cyber-attack. In [42] the problem of selecting the small subsets of measurements that can be made immune to make the whole system immune from data injection attack is solved. Since this problem becomes really complex because of the large size of the electrical grid, a fast greedy algorithm is used for placing secured PMUs. 


\section{Chapter 3: MATHEMATICAL MODEL AND FORMULATION}

\subsection{Theory of Multiple Linear Regression (MLR) [48]}

Multiple linear regression (MLR) is a method used to model the linear relationship between a dependent variable and one or more predictor (independent) variables. The dependent variable can be load, voltage per unit, transmission loss function, etc., and the independent variables are the variables affecting these dependent variables in any way. The regression model expresses the value of a dependent variable as a linear function of one or more predictor variables and an error term.

$Y=b_{0}+b_{1} X_{1}+\cdots+b_{k} X_{k}+e$

Where, $Y$ is the dependent variable, $X_{1}, X_{2}, \ldots, X_{k}$ are the predictor variables, $b_{1}, b_{2}, \ldots ., b_{k}$ are regression parameters with respect to $X_{1}, X_{2}, \ldots, X_{k}$, and $e$ is the error term.

Let each of the $k$ predictor variables, $X_{1}, X_{2}, \ldots ., X_{k}$, have $n$ levels. The system of $n$ equations can be represented in matrix notation as follows:

$\mathrm{Y}=\mathrm{Xb}+\mathrm{e}$

Where

$\mathbf{Y}=\left(\begin{array}{c}Y_{1} \\ Y_{2} \\ \cdots \\ \cdots \\ \cdots \\ Y_{n}\end{array}\right)$

$\mathbf{X}=\left(\begin{array}{rrrrrr}1 & X_{11} & X_{12} & \ldots & X_{1 k} \\ 1 & X_{21} & X_{22} & \ldots & X_{2 k} \\ & & \ldots & & \\ & & \ldots & & \\ 1 & X_{n 1} & X_{n 2} & \ldots & X_{n k}\end{array}\right)$

$\mathbf{b}=\left(\begin{array}{c}b_{0} \\ b_{1} \\ \cdots \\ \cdots \\ \cdots \\ b_{k}\end{array}\right)$

$\mathbf{e}=\left(\begin{array}{c}e_{1} \\ e_{2} \\ \cdots \\ \cdots \\ \cdots \\ e_{n}\end{array}\right)$

The matrix $\mathbf{X}$ contains information about the levels of the predictor variables at which the observations are obtained. The vector $\mathbf{b}$ contains all the regression coefficients. The difference between the actual value of $Y$ and the predicted value $\hat{Y}$ would, on average, tend toward 0 , so it 
can be assumed that the error term in equation (2) has an average value of 0 . The error term can therefore be omitted in calculating parameters.

The estimates of $\mathbf{b}$ are then obtained to get the regression model by using the least squares method such that the sum-of-squares of differences of observed and predicted values is minimized. The estimated $\mathbf{b}$ obtained is

$\hat{b}=\left(X^{\prime} X\right)^{-1} X^{\prime} Y$

The multiple linear regression model also referred as fitted model can now be estimated as:

$\widehat{Y}=\hat{b}_{0}+\hat{b}_{1} X_{1}+\cdots+\hat{b}_{k} X_{k}$

Or in matrix notation: $\hat{Y}=X \hat{b}$

The observations, $Y_{i}$, may be different from the fitted values $\hat{Y}_{i}$ obtained from this model. The difference between these two values is the residual, $\hat{r}_{i}$. The vector of residuals, $\hat{\mathbf{r}}$, is obtained as:

$\hat{r}=Y-\hat{Y}$

The regression equation is estimated such that the total sum-of-squares can be partitioned into components due to regression and residuals:

$S S T=S S R+S S E$

Where

$S S E=\sum_{i=1}^{n}\left(Y_{i}-\hat{Y}_{i}\right)^{2} \quad$ sum of squares, error

$S S T=\sum_{i=1}^{n}\left(Y_{i}-\bar{Y}\right)^{2} \quad$ sum of squares, total

$S S R=\sum_{i=1}^{n}\left(\hat{Y}_{i}-\bar{Y}\right)^{2} \quad$ sum of squares, regression

$\bar{Y}$ : average value of $Y_{i}$

The explanatory power of the regression is explained by its $R^{2}$ value, calculated from the sumsof-squares terms as 
$R^{2}=\frac{S S R}{S S T}=1-\frac{S S E}{S S T} \in[0,1]$

The residual mean square (MSE) is the sample estimate of the variance of the regression residuals.

$M S E=\frac{S S E}{n-k-1}$

The $R^{2}$ value and $M S E$ value are the two goodness of fit of measurements which explains how well the predictor variables explain the dependent variable. The closer the $R^{2}$ value is to 1 and the smaller the $M S E$ value, the better the estimated regression function fits the data.

\subsection{Modeling attacks and anomalies}

There are two attacks considered here the data integrity and LR attack as well as inconsistencies in electrical design parameters. This section models both the attacks and parameter inconsistencies.

\subsubsection{Inconsistencies in electrical design parameters}

A distribution system model has many parameters which define it completely like line resistance $(\mathrm{R})$, line reactance $(\mathrm{X})$, line geometry, conductor type, regulators type, etc. Any incorrect or uninformed parameter will lead to model errors and hence risk of operation of the entire system. Here inconsistencies are modeled as statistical variations with probabilities such as for example a variation $\delta \mathrm{X}$ in a distribution line $\mathrm{L}$ with reactance $\mathrm{X}$.

\subsubsection{Data integrity attack [29]}

This attack often requires detailed knowledge of the communication protocols to send false or malicious status or control signals. [44] presents a data integrity attack on control signals of voltage control of FACTs devices and substation controllers. Similar attack on a voltage control loop of a distribution system is considered where the attacker gained information of a few critical capacitors. 
If $u(t)$ is the actual signal from the control center and $\left[u_{\min }(t), u_{\max }(t)\right]$ is the range of possible control signals. Let us define "Min attack" as an attack where $u(t)=u_{\min }(t)$ for $t \in \tau_{a}$ the duration of attack and a "Max attack" as an attack where $u(t)=u_{\max }(t)$. Such a min-max attack is unobservable as presented in [29] and its effect is more profound in the region near the impact.

\subsubsection{Load redistribution attack}

This attack is a subclass of false data injection attack where the load changes by $L+\Delta L$ at some buses and L $-\Delta \mathrm{L}$ at other buses while the total load remains unchanged. It is assumed here that only load bus power injection measurements and line power flow measurements can be attacked and that physically protecting all the meters is not feasible. Also it has been proved theoretically in [35] that LR attacks cannot be detected by any of the existing techniques for bad data detection in a state estimator. However, since short-term load forecasting provides an approximate estimation of the load, an attack that results in larger $\Delta \mathrm{L}$ may be detected so for an undetectable attack, $\tau<0.5 \mathrm{~L}$ and $\sum L+\Delta L=L$ where $\tau$ is the load multiplier. So in our work, we suppose that the attack magnitude for a load measurement does not exceed $\tau=50 \%$ of its true load value.

The essential conditions for creating undetectable load redistribution (LR) attacks are proposed in [35]. For an undetectable load redistribution attack the total loading in the system should remain the same i.e. $\sum L+\Delta L=L$. It is also essential that the change in load at each node should remain within an acceptable limit which can be maintained by keeping $\tau<0.5 \mathrm{~L}$ where $\tau$ is a load multiplier. 


\subsection{Distributed Cyber Attack Detection Method}

Consider a power system with a set of buses $\lambda$, a set of buses $L \subset \lambda$ with known demand, a set of buses $S \subset \lambda$ with solar generating units and a bus $C \subset \lambda$ with a capacitor unit. Let the voltage of a bus $\mathrm{j} \in \mathrm{J}$ a set of $\{1,2, \ldots, \lambda\}$ buses, be given by $V p u_{j}$ where each element is a k-dimensional real vector from $\mathrm{k}$ network conditions. A k-means clustering as in eq. (10) is employed where $\subset$

$V p u_{\mu_{i}}=\frac{1}{h * k} \sum_{i=1}^{k}\left(V p u_{a_{i}}+V p u_{b_{i}}+\cdots+V p u_{h_{i}}\right)$

Cluster set $\mathrm{S}=\left\{S_{1}, S_{2}, \ldots, S_{t}\right\}$ are assigned to datapoints where $S_{i}$ is the set of observations \{ $\left.V p u_{a}, V p u_{b}, \ldots ., V p u_{h}\right\}$ and $\mathrm{h} \subset \lambda$.

An agent $A_{j}$ from a cluster $S_{j}$ is

$$
\min _{i} \sum_{i=1}^{k}\left|\left(V p u_{j}-V p u_{\mu_{i}}\right)\right| \forall j=a, b, \ldots, h
$$

From a highly correlated set of agents $A_{i}$ and $A_{j}$ an agent pair is created where $A_{j} \in S_{j} \cap A_{i} \in S_{i}$ $=\phi$ and $S_{i}$ and $S_{j} \subset \mathrm{S}$. Consider the behavior of agent $A_{i}$ on $V p u_{j}$ as a regressive model given by eq. (12).

$V p u_{m}(k)=b_{0}+b_{1} V p u_{n}(k)+e_{1}(k)$

The calculation of the coefficients $b_{i}$ result in eq. (13)

$\widehat{V} p u_{m}=\hat{b}_{0}+\hat{b}_{1} V p u_{n}$

Similarly, the statistical relationships between all the selected agent pairs are declared as shown in figure 3. A distribution network under cyber-attack with the bus voltages of the elected pair is compared to corresponding bus voltages in (13) obtained from the same network. If the variation exceeds a certain limit, the agent declares a cyber-attack event. A consensus of at least two agent pairs results in a final decision of cyber-attack event. 


\subsection{Proposed Voltage Controller Methodology}

Consider a power system with a set of buses $\lambda$, a set of buses $L \subset \lambda$ with known demand, a set of buses $\mathrm{S} \subset \lambda$ with solar generating units and a bus $\mathrm{C} \subset \lambda$ with a capacitor unit. Let total combined real power demand at buses $L$ be $d$, the real power generated at bus $S$ be $p$, the total $\mathrm{kV}$ ars injected by the capacitor at bus $C$ be $K v a r$, and the voltage per unit at a bus $j$ from set $\lambda$ is $V p u_{j}$. The variables of total loading of the system, the real power output of the DG connected and the reactive power of the capacitor having a correlation with node voltage $\mathrm{j}$ are chosen as predictor variables. The dependent variable is the voltage in p.u. (Vpu) at any bus $\mathrm{j}$ violating voltage limits.

A statistical model of dependent variable, with defined predictor variables is given by eq. (14).

$\operatorname{Vpu}_{j}(k)=b_{0}+b_{1} d(k)+b_{2} p(k)+b_{3} K \operatorname{var}(k)+e(k)$

A calculation of coefficients of $b_{i}$ yields eq. (15) for $\mathrm{Vpu}$ of a bus $\mathrm{j}$ which is further used for voltage control.

$\widehat{V} p u_{j}=\hat{b}_{0}+\widehat{b}_{1} d+\widehat{b}_{2} p+\hat{b}_{3} K v a r$

Similarly, statistical models are developed for all the buses prone to voltage violations in the distribution system. Once the statistical models are obtained from Minitab software for the chosen distribution system, statistical reactive power model algorithm for calculation of $\mathrm{kVars}$ required to bring the voltage of the violated buses within the permissible limits is developed in Matlab. The inputs for the Kvar calculation model are the statistical models from eq. (15) and the required voltage at the violated bus and the output is the estimated $\mathrm{kVars}$ required to correct the voltage violations in that bus as shown in figure 8.

The estimated $\mathrm{kVars}$ obtained from this algorithm is not affected by data injection attacks till the network topology changes as the statistical models from eq. (15) are obtained from validated data sets of the dependent and predictor variables. 


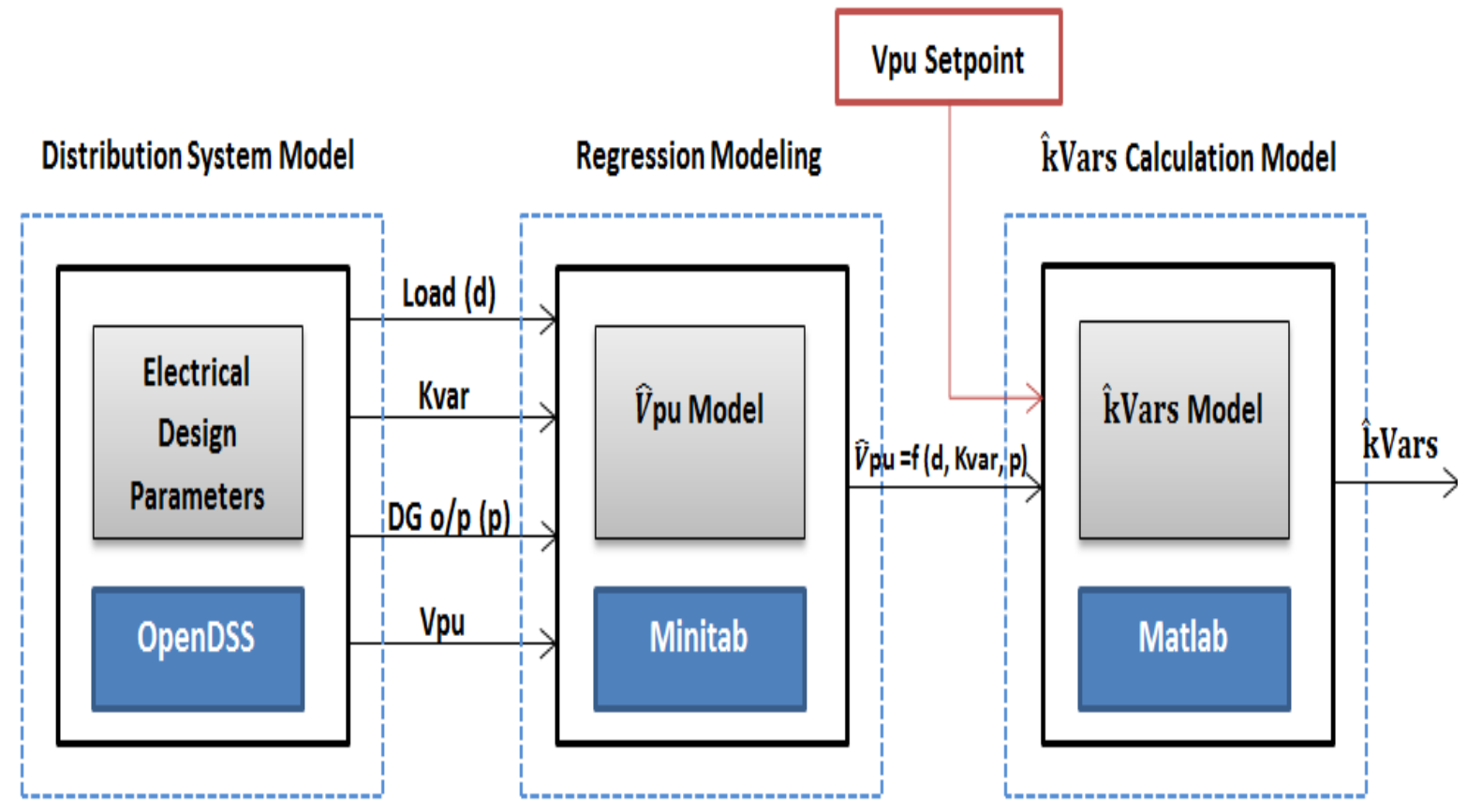

Figure 8 A Statistical Reactive Power Model Algorithm 


\section{Chapter 4: SIMULATION TOOLS AND SOFTWARE}

This chapter briefly describes various software packages used in this thesis for modeling the electric distribution system, to make the voltage controller models and study their effects under different abnormal conditions and cyber-attack detection models.

Out of the various commercially available simulation tools for modeling utility distribution systems we chose OpenDSS for doing distribution power flow in this thesis. The other software tools were CYMDIST, Milsoft, Windmil, etc. The voltage controller models are developed in MATLAB with the distribution network of OpenDSS being interfaced with it through COM Interface.

The multiple linear regressions among the chosen dependent and independent variables for developing the voltage controller models and cyber-attack detection models are made in Minitab software. Other software used for doing regression analysis is STATA, data analysis package of MS Excel, etc. but we chose Minitab for its excellent representation of the results. The tools used in this research work are described with their abilities and their detailed importance in the following sections.

\subsection{Open Distribution System Simulator (OpenDSS)}

The Open Distribution System Simulator (OpenDSS or simply DSS) is an all-inclusive electrical system simulation tool for electric utility distribution systems. OpenDSS is open source software developed by the Electric Power Research Institute [45]. It can be used to steady state analysis, integration of distributed generation and time series power flow. It has also developed various test cases for all IEEE benchmark test feeders starting from IEEE 4 node to IEEE 8500 node test feeders. It provides two implementations; first one is a standalone executable platform and the other one is through COM server DLL, which can be used to drive OpenDSS from a myriad of other platforms. The executable version makes use of basic direct script codes through which user can develop circuits and solve them. The COM interface enables the user to drive OpenDSS externally from any third party analysis programs like MATLAB, VBA, C\#, Pyhton etc. and 
execute custom solution modes and features of the simulator. The DSS is designed in such a way so that it can to be effortlessly altered to meet future needs.

The OpenDSS program can be used for the following applications [45]:

- Distribution Planning and Analysis

- $\quad$ General Multi-phase AC Circuit Analysis

- $\quad$ Analysis of Distributed Generation Interconnections

- $\quad$ Annual Load and Generation Simulations

- $\quad$ Risk-based Distribution Planning Studies

- Neutral-to-earth Voltage Simulations

- Solar PV System Simulation

- Wind Plant Simulations

- $\quad$ Storage Modeling

- Distribution Feeder Simulation with AMI Data

- Distribution State Estimation

- $\quad$ EV Impacts Simulations

- Analysis of Unusual Transformer Configurations

OpenDSS configuration is shown in figure 9 [46] which show the three different ways by which the DSS engine can be initiated.

- $\quad$ OpenDSS scripts - Using direct scripting codes to define the circuit and solve it.

- $\quad$ COM interface - Driving it externally from any third party analysis program.

- User Written DLL - Writing suitable DLL which can be linked with the engine. 


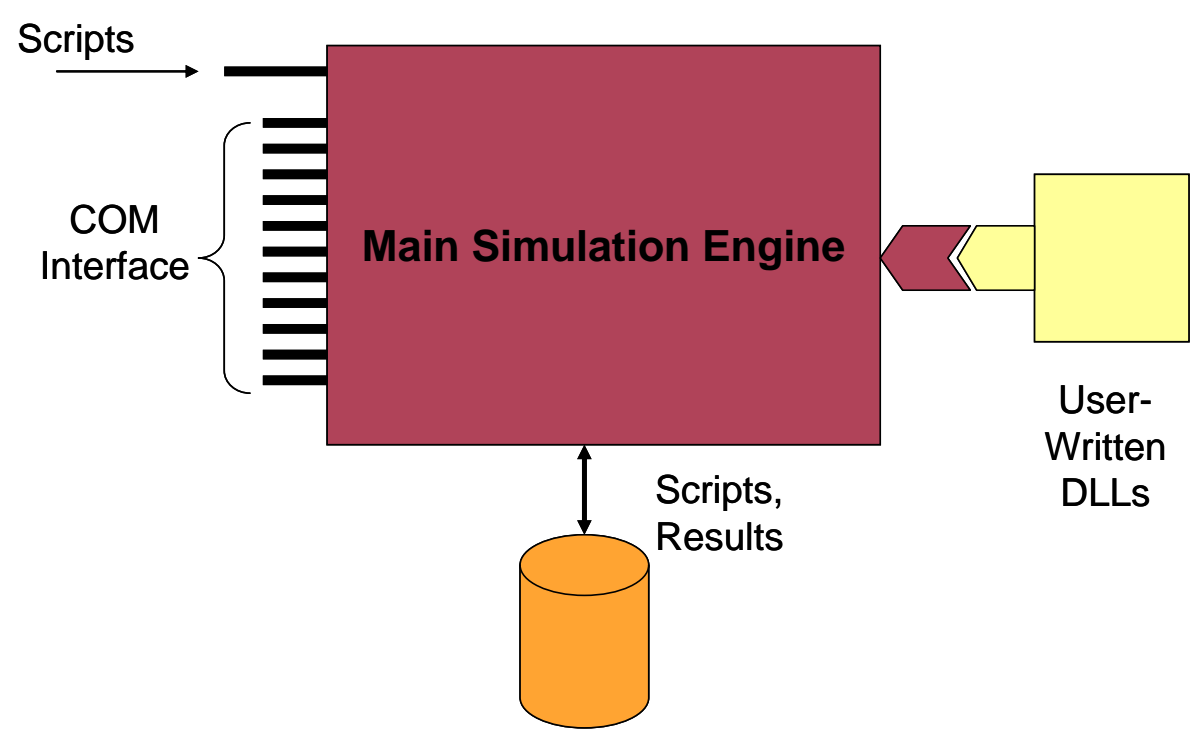

Figure 9 OpenDSS Configuration [46]

Some of the special features of OpenDSS used in this work are listed below.

\subsubsection{Extensive Range of Solution Modes}

A number of solution applications are available in OpenDSS. OpenDSS can solve a basic power flow for a distribution system in which the substation is modeled as an infinite source of energy. The two methods used to solve power flow in DSS are the iterative and direct power flow methods. For iterative power flow method, the loads and distributed generators are modeled as injection sources. The algorithms used to solve power flow problem are the normal current injection and Newton current injection. For the direct mode, the loads and generators are included in system admittance matrix as admittances and this mode is solved directly without any iteration.

DSS can solve both the meshed systems and radial systems with equal ease. The snapshot power flow mode does a single power flow solution at the current load. Similarly, daily, yearly and duty cycle power flow mode are also available for simulations of different periods. User specified load shape or a default load shape by the engine can be used for a daily, yearly or duty cycle power flow mode. 
After solving the power flows, losses, voltages, currents and other data are accessible for the feeder system. The losses in terms of $\mathrm{kW}$ or $\mathrm{kVar}$ losses for each time instant for all the zones and loads are provided by the energy meters. A three phase unbalanced distribution power flow for the AEP feeder is performed using OpenDSS in this thesis. Also a PV unit and a capacitor bank are connected for the calculation of voltage controller in OpenDSS and the power flows are calculated.

\subsubsection{COM Interface}

The most useful feature of OpenDSS is the COM interface which allows user to execute custom solutions modes from an external platform and drive the DSS simulator from that platform and perform various analyses that cannot be done using the direct script codes. The third party programs are MS Office tool through VBA, MATLAB, Python, C\# etc. Also through COM interface user can do solve loops like for, if, if then else etc. which are not available in the direct scripts in DSS. Most of the results of the DSS engine can also be retrieved through the COM interface.

This Interface is used to drive the OpenDSS through MATLAB and calculate the required $\mathrm{kVars}$ from the capacitor bank for different network conditions and line losses for different geometries in the distribution networks for the study in our thesis.

\subsection{Minitab}

Minitab is a statistics package developed at the Pennsylvania State University by researchers Barbara F. Ryan, Thomas A. Ryan, Jr., and Brian L. Joiner in 1972. It can be used to do various types of regression analysis like linear regression, non-linear regression, and orthogonal regression. It is also used for doing time series plots, developing ANOVA tables, correlation analysis, cascading of graphs and various other functions.

Minitab is used in our thesis to do the regression analysis for the voltage controller models and the cyber-attack detection models. It is also used to present the results in a more accurate way. 


\section{Chapter 5: SIMULATION AND RESULTS}

This chapter discusses all the results of this research by introducing the test systems used and the algorithms simulated on these test systems. Also, the behavior of the test systems are studied through voltage and loss analysis results. Interpretations are drawn from these resultant plots to back the proposed ideas and goals of this thesis work.

\subsection{3-Bus Distribution System}

A 13-bus distribution system, shown in figure 10, is used to illustrate the proposed voltage controller based on MLR technique. System parameters include:

- total load of $3466 \mathrm{~kW}$ and $2102 \mathrm{kvar}$

- two 600-kvar capacitor banks (bus 675 and bus 611)

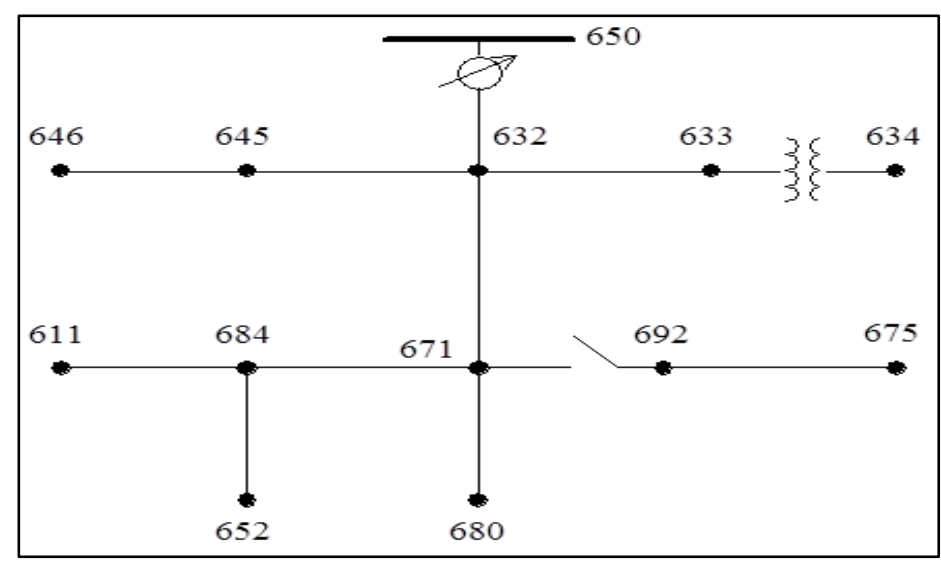

Figure 10 IEEE 13 Bus Distribution Test Feeder

The regulator controls are turned off to enhance the effects the capacitor control, and a 3-phase solar generating unit is added at bus 632. Power flow is run on the system for different loading and DG power output. Voltages of two buses, bus 652 and bus 684 violated the permissible limits. A 2-phase capacitor is added at bus 684 which is a logical choice as the bus 684 violated the voltage limits. Regression models are developed, taking the voltage in p.u.at each bus as the dependent variable in each model. A data set of 36 observations is generated with the OpenDSS for some randomly selected values of predictor variables and the correspondingly obtained 
dependent variable. The models are regressed and the coefficient of determination $R^{2}$, and the mean squared error $M S E$ are calculated.

Table 1 show the regression models obtained for bus 652 and 684 and their corresponding $R^{2}$ and MSE values. It can be seen that $\mathrm{R}^{2}$ values are close to one and MSE values are close to zero for all the models showing a good fit and proving that the predictor variables are able to explain almost $100 \%$ variations in the dependent variable. Moreover, the normal probability plot of the residual for the regression models shown in figure 11 also shows that most of the red points are clustered around blue line indicating that the error terms are approximately normal indicating the goodness of fit of all the models.

Table 1. Regression Models for Voltage in p.u. for Buses 652 and 684

\begin{tabular}{llll}
\hline Bus & Regression Model & $\boldsymbol{R}^{\mathbf{2}}$ & $\boldsymbol{M S E}$ \\
\hline 652 & $1.014169-0.09631 * d+5.62 \mathrm{e}-06^{*} p+6.77 \mathrm{e}-05^{*}$ Kvar & 0.9957 & $2.79 \mathrm{e}-05$ \\
684 & $1.014493-0.09142 * d+5.54 \mathrm{e}-06^{*} p+6.8 \mathrm{e}-05^{*}$ Kvar & 0.9977 & $2.74 \mathrm{e}-06$ \\
\hline
\end{tabular}

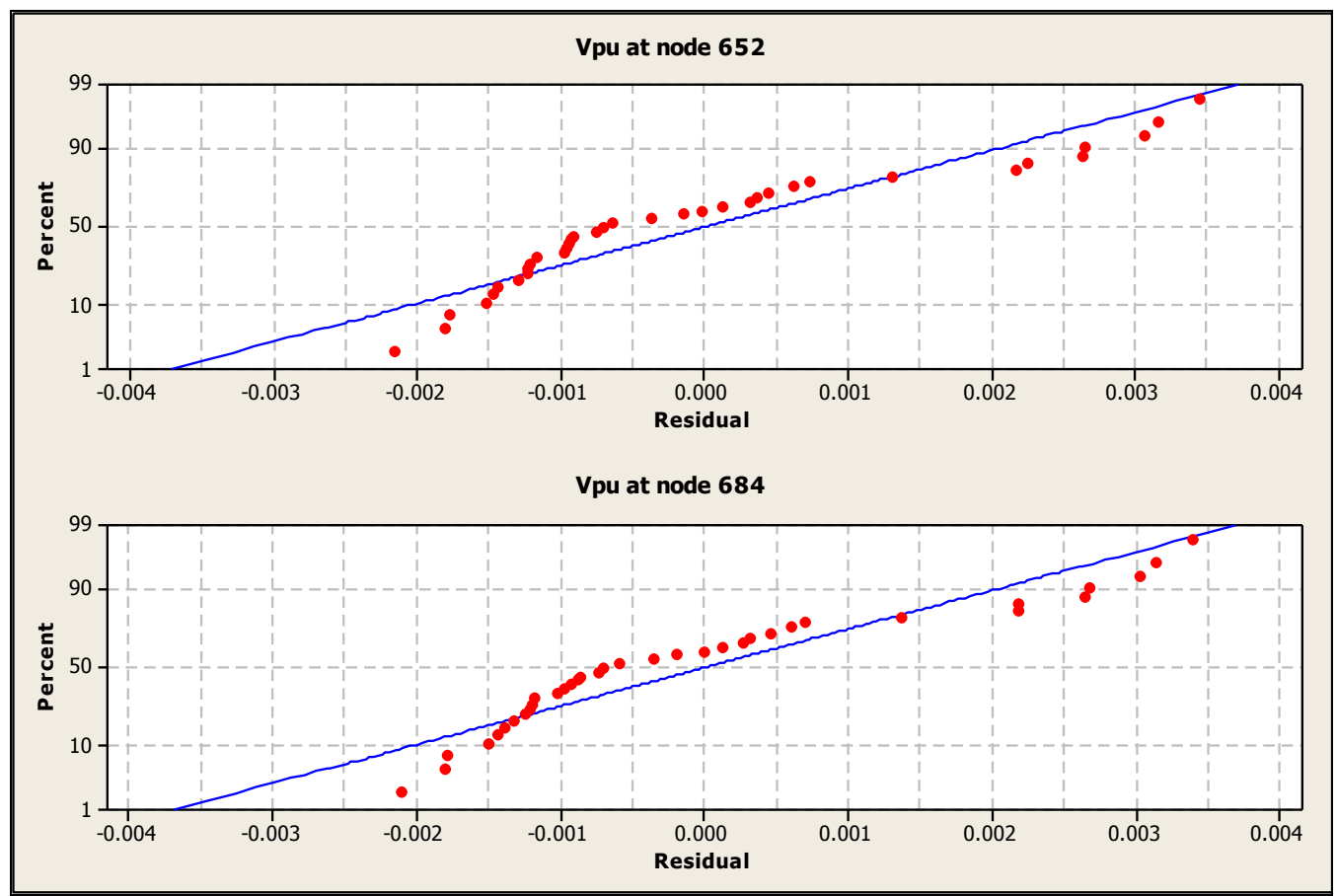

Figure 11 Normal Probability Plots for the Regression Models for Buses 652 and 684 
The Kvars calculation models (Model 2) developed in Matlab from the statistical reactive power model algorithm are validated for some random values of loading and real power output of the PV unit as shown in table 2. The Kvars calculated from Model 2 are compared with the Kvars obtained from a conventional capacitor controller (Model 1) in OpenDSS to bring the voltage in $\mathrm{pu}$ at the violating buses to $0.95 \mathrm{pu}$. It is observed that the Kvars values from both the models are really close.

Table 2. Controller Validation for the $\mathrm{kVar}$ Calculation Models

\begin{tabular}{lllllll}
\hline \multirow{2}{*}{ Load } & $\begin{array}{l}\text { PV output } \\
(\mathbf{k W})\end{array}$ & $\begin{array}{l}\text { Vpu } \\
\text { Required }\end{array}$ & & \multicolumn{2}{l}{ kVars (652) } & \multicolumn{2}{c}{ kVars (684) } \\
\cline { 4 - 6 } & & & Model 1 & Model 2 & Model 1 & Model 2 \\
\hline 1.205 & 0 & 0.95 & 788 & 766.4 & 691 & 671.6 \\
1.401 & 8 & 0.95 & 1055 & 1044.6 & 942 & 934.4 \\
1.058 & 520 & 0.95 & 539 & 514.1 & 456 & 431.6 \\
1.208 & 480 & 0.95 & 752 & 730.8 & 655 & 636.5 \\
1.352 & 120 & 0.95 & 978 & 965.5 & 870 & 859.4 \\
0.975 & 0 & 0.95 & 468 & 439.2 & 391 & 362.4 \\
\hline
\end{tabular}

Simulation results on a real-world, large-scale distribution system is presented next. The accuracy of the proposed voltage controller based on MLR technique is demonstrated, its robustness with inconsistencies in electrical design parameters and cyber-attack conditions is also shown. The accuracy of the proposed distributed cyber-attack detection technique is also validated for both the cyber-attacks. Specifically, this section will present the following:

\subsection{AEP Test Circuit}

In previous research work co-authors [47] have modeled AEP system and studied impact of PV penetration. The AEP feeder I, shown in figure 12, is radial 395 buses system and is fed by a 12.47 KV medium voltage substation modeled as a voltage source behind impedance. The distribution system includes two main circuits with laterals and distributed loads. The aggregated loads represent a mixture of residential and industrial loads and the total load on the system is 
2.27 MVA (2.042 MW and 1.00 MVAR) and the active power losses represent $1.52 \%$ of the total system load. The load buses in this system are modeled as PQ loads. Three voltage regulators (two 3-phases and one single phase) employed in this feeder are turned off. A 3-phase solar power generating unit is added at bus 43_west and a 1-phase capacitor is added at bus 131_west.3.

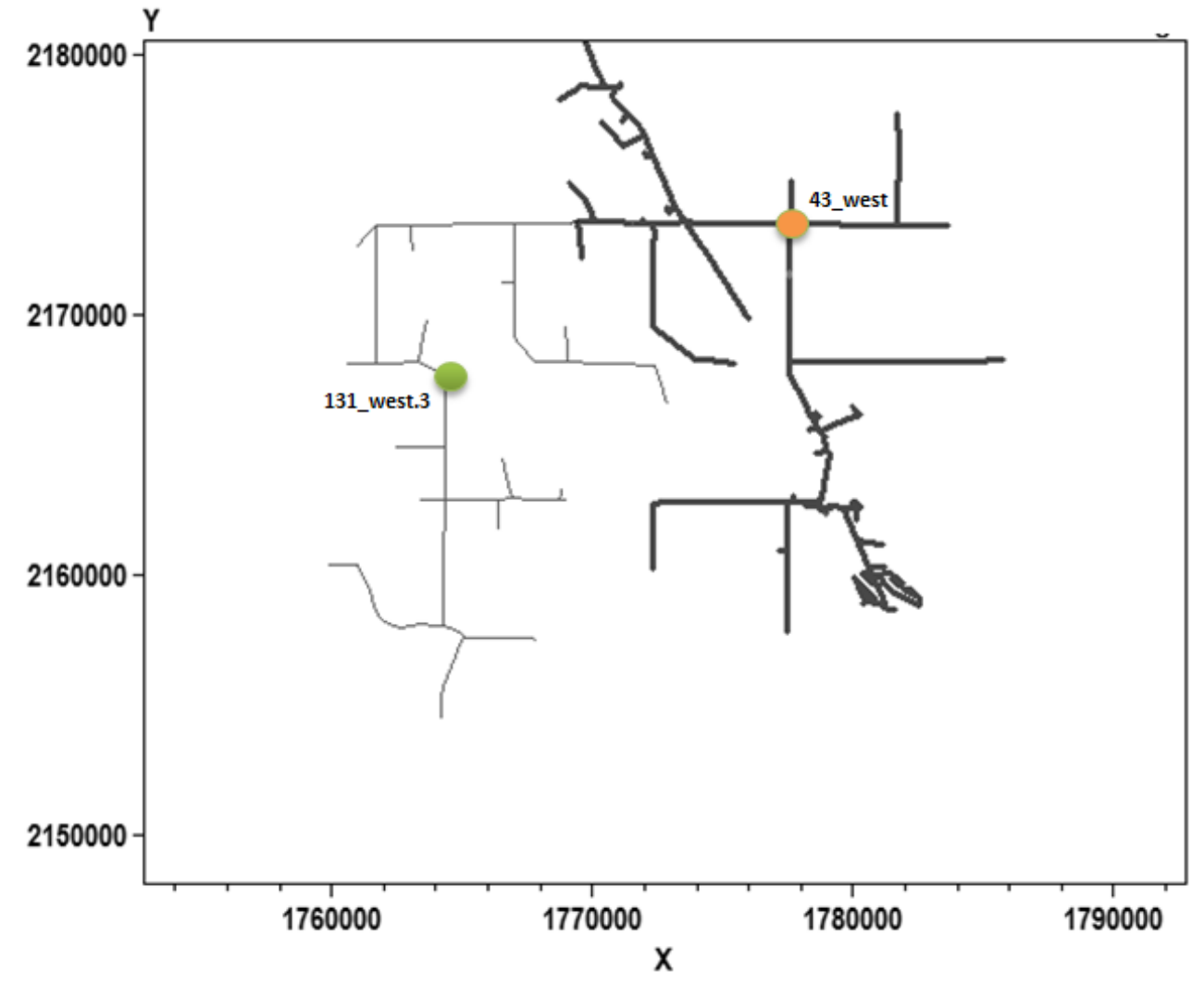

Figure 12 AEP Feeder 1 Network Diagram

\subsubsection{MLR based Statistical Voltage Controller}

A an unbalanced three-phase power flow (UTDPF) is performed to obtain voltage profile of the distribution system. The active power generation of the renewable generators in the system is increased according to their daily generation curve and a power flow solution is again performed to obtain the voltages of the buses. Starting from the substation the sections where the voltage is most frequently violated for different network conditions are identified. These sections also form a logical choice for location of capacitors. With variations in active power generation, four buses 
164_west, 146_west, 143_west and 132_west have the most voltage violations and are therefore considered as dependent variables. The predictor variables here are the kVars from the capacitors, active power output of PV, the total loading and the models are defined in table 3. The coefficient of determination $\left(R^{2}\right)$ and the mean squared error (MSE) as well as the plot of the residual of datapoints vs the model show the accuracy of the models.

It can be seen from table 3 that $\mathbf{R}^{2}$ values are close to one and MSE values are close to zero for all the models showing a good fit and proving that the predictor variables are able to explain almost $100 \%$ variations in the dependent variable. Figure 13 shows the normal probability plot of the residual for the regression models and indicated the goodness of fit of all the models.

Table 3. Regression Models for Voltage in p.u. for Buses 164_west, 146_west, 143_west and 132_west.

\begin{tabular}{|c|c|c|c|}
\hline Bus & Regression Model & $\mathbf{R}^{2}$ & MSE \\
\hline 164_west & $1.021319-0.09819 * d+1.13 \mathrm{e}-05^{*} p+0.000202 * K v a r$ & 0.9995 & $5.2 \mathrm{e}-07$ \\
\hline 143_west & $1.021469-0.09569 * d+1.13 \mathrm{e}-05 * p+0.000202 * K v a r$ & 0.9994 & $6.25 \mathrm{e}-07$ \\
\hline 146_west & $1.021413-0.0971 * d+1.13 \mathrm{e}-05 * p+0.000202 *$ Kvar & 0.9995 & $5.53 \mathrm{e}-07$ \\
\hline 132_west & $1.020864-0.09159 * d+1.16 \mathrm{e}-05 * p+0.000201 * K v a r$ & 0.9991 & $9.57 \mathrm{e}-07$ \\
\hline
\end{tabular}



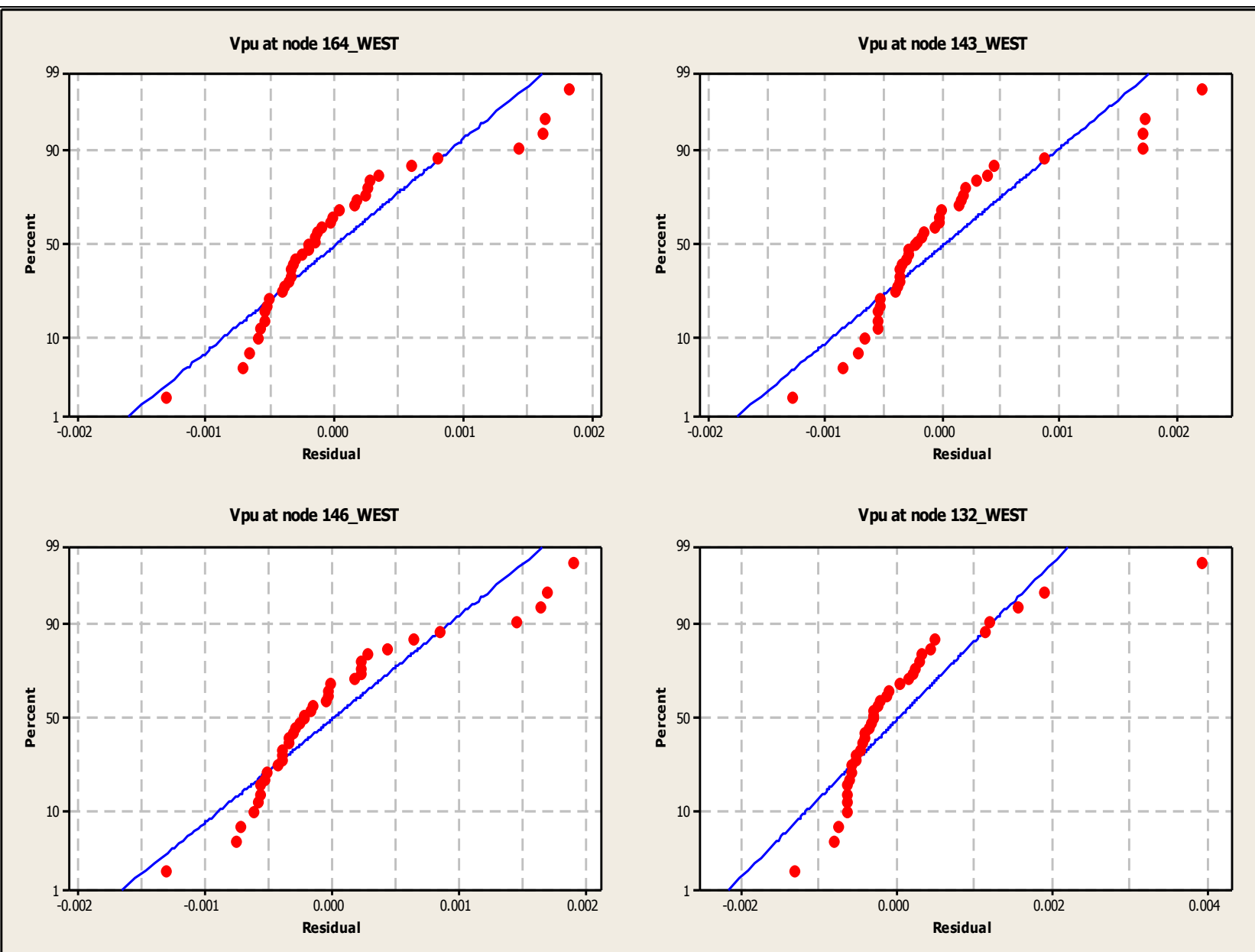

Figure 13 Normal Probability Plots for the Regression Models for Buses 164_west, 146_west, 143_west and 132_west

The reactive power outputs from the regressive model (Model 2) for a typical 24 hour load curve with a peak load of $1.42 \mathrm{KW}$ are shown in figure 14. These reactive power requirements are compared with those obtained from a traditional capacitor control achieved in OpenDSS (Model 1) and as seen in figure 14. 


\begin{tabular}{|c|c|c|c|c|c|c|c|c|c|c|c|}
\hline \multirow{2}{*}{ Hours } & \multirow{2}{*}{\multicolumn{2}{|c|}{ Load }} & \multirow{2}{*}{ PV Power Output } & \multicolumn{2}{|c|}{ Kvar (164_WEST) } & \multicolumn{2}{|c|}{ Kvar (143_WEST) } & \multicolumn{2}{|c|}{ Kvar (146_WEST) } & \multicolumn{2}{|c|}{ Kvar (132_WEST) } \\
\hline & & & & Model 1 & Model 2 & Model 1 & Model 2 & Model 1 & Model2 & Model 1 & Model 2 \\
\hline 1 & & $85 \%$ & $0 \%$ & 234.5 & 232.7 & 219 & 217 & 227.5 & 225.7 & 198.5 & 196.5 \\
\hline 2 & & $85 \%$ & $0 \%$ & 234.5 & 232.7 & 219 & 217 & \begin{tabular}{|l|l|}
7 & 227.5 \\
\end{tabular} & 225.7 & 198.5 & 196.5 \\
\hline 3 & & $85 \%$ & $0 \%$ & 237 & 235.1 & 221 & 219.4 & 230 & 228.1 & 201 & 198.8 \\
\hline 4 & & $86 \%$ & $0 \%$ & 244.5 & 242.4 & 228.5 & 226.5 & 237.5 & 235.3 & 208 & 205.6 \\
\hline 5 & & $99 \%$ & $1 \%$ & 332.5 & 327.6 & 314 & 309.5 & 324.5 & 319.6 & 290.5 & 285.5 \\
\hline 6 & & $100 \%$ & $5 \%$ & 341 & 335.5 & 322 & 317.2 & 332.5 & \begin{tabular}{|l}
327.4 \\
\end{tabular} & 298 & 292.8 \\
\hline 7 & & $95 \%$ & $15 \%$ & 302.5 & 299.1 & 280.5 & 281.6 & 295 & 291.3 & 262 & 258.3 \\
\hline 8 & & $95 \%$ & $40 \%$ & 289.5 & 286.3 & 271.5 & 269 & 281.5 & 278.6 & 249 & 245.6 \\
\hline 9 & & $91 \%$ & $52 \%$ & 261 & 259 & 244 & 242.2 & 253.5 & 251.5 & 222 & 219.5 \\
\hline 10 & & $85 \%$ & $60 \%$ & 215.5 & 214 & 199.5 & 198.3 & 208.5 & 207 & 179 & 177.1 \\
\hline 11 & & $79 \%$ & $65 \%$ & 175 & 173.4 & 160 & 158.7 & 168.5 & 166.9 & 141 & 138.9 \\
\hline 12 & & $79 \%$ & $68 \%$ & 174 & 172.4 & 159 & 157.7 & 167.5 & 165.9 & 140 & 137.9 \\
\hline 13 & & $75 \%$ & $65 \%$ & 140 & 139.4 & 126.5 & 125.6 & 134 & 133.2 & 108.5 & 107 \\
\hline 14 & & $63 \%$ & $61 \%$ & 66.5 & 62.5 & 54.5 & 50.6 & 61 & 57.2 & 39.5 & 35.1 \\
\hline 15 & & $56 \%$ & $48 \%$ & 20.5 & 14.8 & 10.5 & 4.3 & 16 & 10.1 & 0 & 0 \\
\hline 16 & & $55 \%$ & $35 \%$ & 20 & 14.3 & 10 & 4 & 15.5 & 9.7 & 0 & 0 \\
\hline 17 & & $49 \%$ & $10 \%$ & 0 & 0 & 0 & 0 & 0 & 0 & 0 & 0 \\
\hline 18 & & $56 \%$ & $1 \%$ & 38 & 33 & 27.5 & 22.5 & 33.5 & 28.3 & 14 & 9.3 \\
\hline 19 & & $56 \%$ & $0 \%$ & 38.5 & 33.4 & 28 & 22.8 & 34 & 28.6 & 14.5 & 9.7 \\
\hline 20 & & $69 \%$ & $0 \%$ & 123 & 120.9 & 110 & 108.1 & 117.5 & 115.1 & 94 & 91.7 \\
\hline 21 & & $54 \%$ & $0 \%$ & 26.5 & 21.2 & 16.5 & 11 & 22 & 16.6 & 3.5 & 0 \\
\hline 22 & & $80 \%$ & $0 \%$ & 199.5 & \begin{tabular}{|l}
197.7 \\
\end{tabular} & 184.5 & 182.9 & 192.5 & \begin{tabular}{|l|}
191.1 \\
\end{tabular} & 165.5 & 163.7 \\
\hline 23 & & $88 \%$ & $0 \%$ & 260 & 257.5 & 243.5 & 241.2 & 252.5 & 250.2 & 222 & 219.8 \\
\hline 24 & & $96 \%$ & $0 \%$ & 311 & 307 & 293 & 289.5 & 303 & \begin{tabular}{|l|}
299.3 \\
\end{tabular} & 270 & 266.2 \\
\hline
\end{tabular}

Figure 14 Controller validation for the Kvar calculation models for buses 164_west, 146_west, 143_west and 132_west 


\subsubsection{MLR based Controller Performance}

In this section, the MLR controller is tested for model consistencies and cyber-attacks. Controller performance under model inconsistency in line of increase in reactance from $1.79 \mathrm{pu}$ to $3.79 \mathrm{pu}$ is considered. Also deception attack is simulated by manipulating the reactive power injection control signal of the capacitor at 131_west from $150 \mathrm{kVar}$ to $300 \mathrm{kVar}(50 \%)$. An LR attack is simulated by manipulating the load value at 644 -7231501-1 from $75 \mathrm{~kW}$ to $150 \mathrm{~kW}$ and decreasing the load value at 382_7231503-1 from $22.5 \mathrm{~kW}$ to $147.5 \mathrm{~kW}$. It should be noted that these events are not simulated simultaneously.

Line Geometry (an example to show the effect of inconsistency of electrical design parameters)

AEP feeder 1 has both overhead lines and underground cables giving away the line losses, but the considerable amount of the losses are given by the overhead lines. Overhead lines have 3 different types of lines having different types of geometries, 2 conductor type, 3 conductor type and 4 conductor type. The 2 conductor type and 4 conductor types, having 17 and 14 different types of line geometries present respectively, are the maximum losses giving lines. For this work, a unity load is taken and no DG is added to the system.

Figure 15 and 16 show the losses on four separate, maximum losses giving lines of a 2 conductor type line and 4 conductor type lines, respectively. The bars with white dots are the lines losses with their original line geometry whereas the remaining of the bars for that line is when other line geometries are used to calculate the losses in those lines. It is observed that line losses are dissimilar for different line geometries used for a same line and this type of inconsistency of an electrical design parameter can result in a catastrophe if we use the results of these for solving some problem related to a distribution system. 


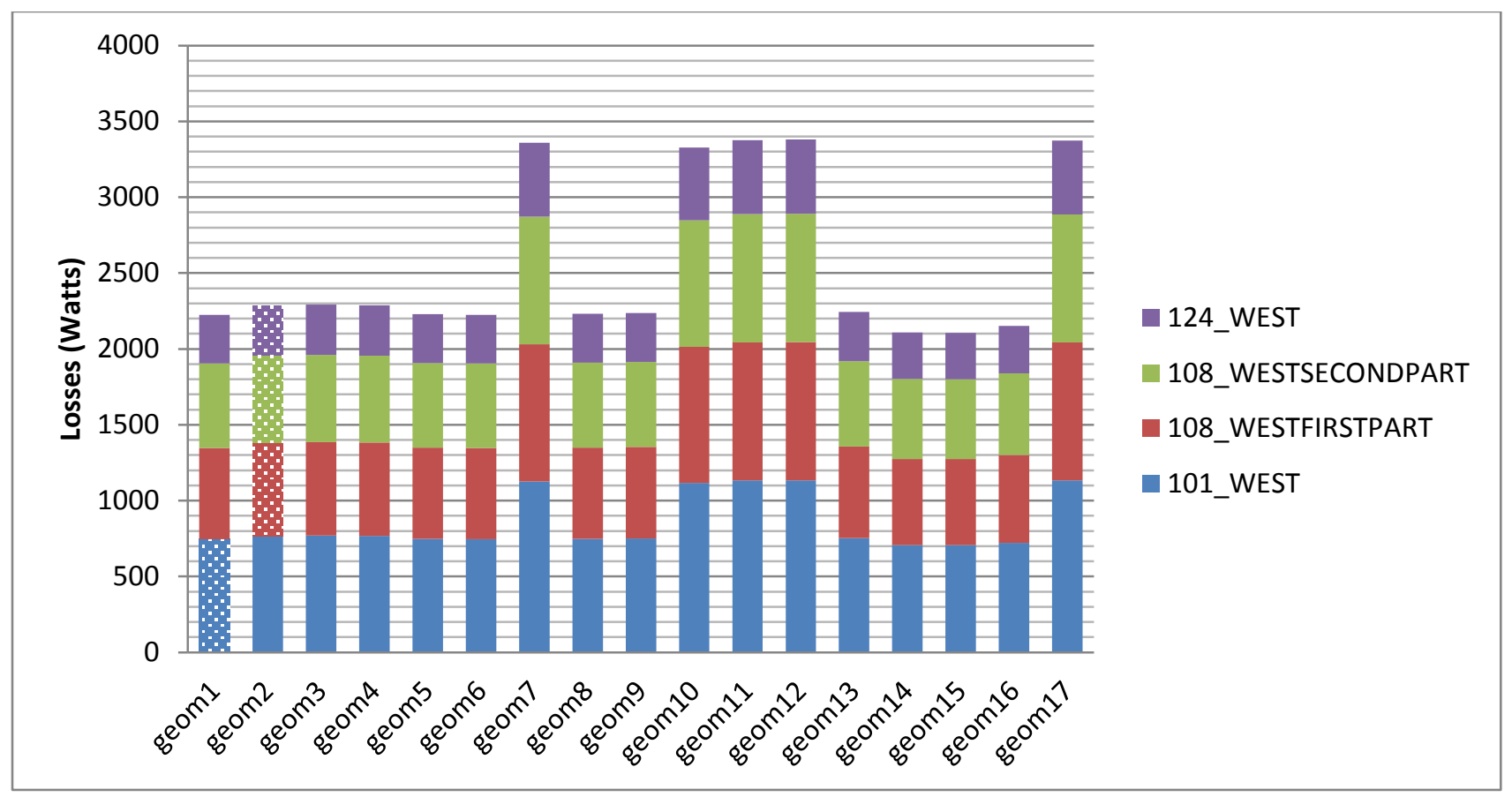

Figure 15 Losses in Four Maximum Losses giving Lines with Each Type of Line Geometry for 2-Conductor Type Line

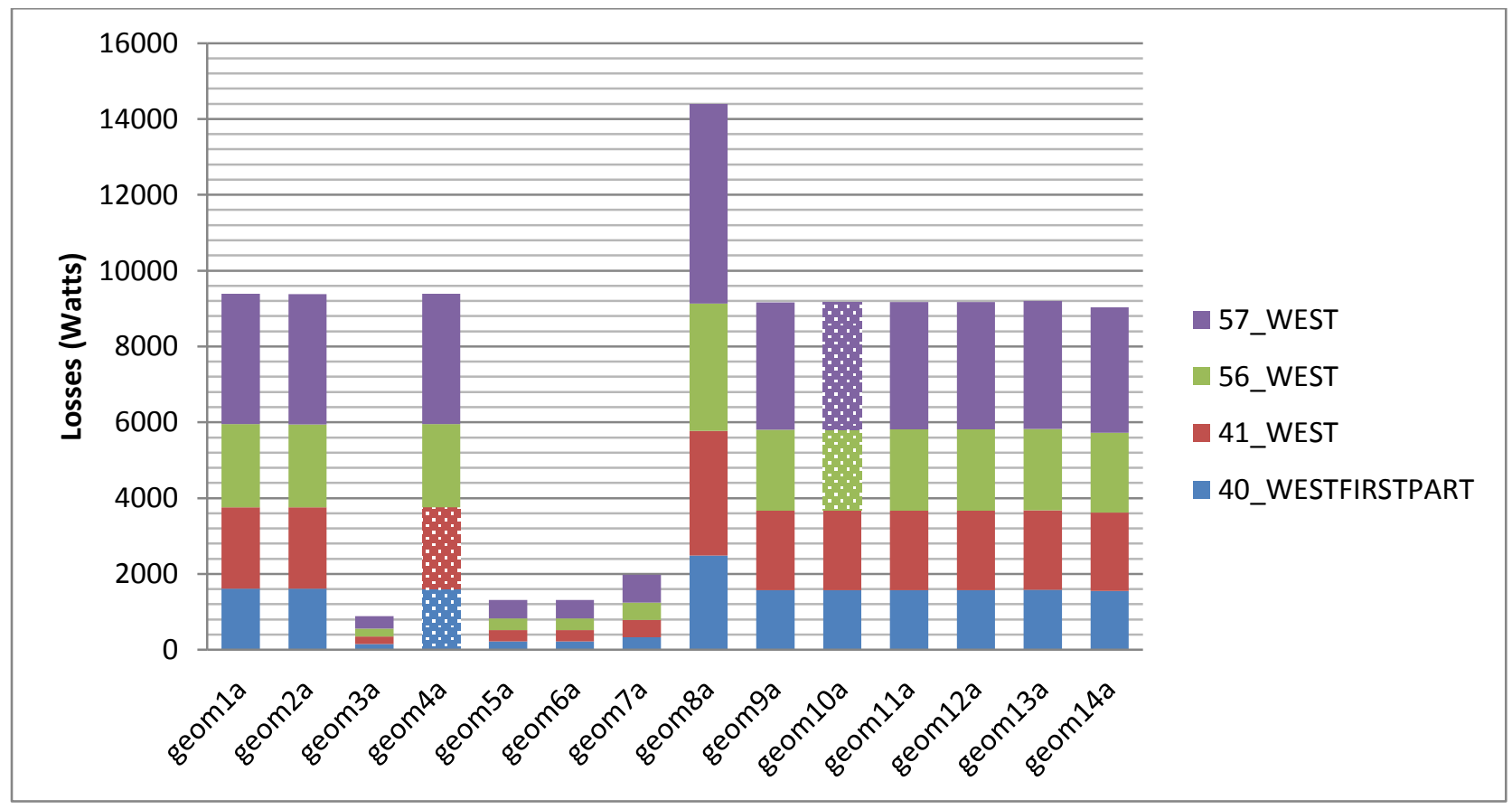

Figure 16 Losses in Four Maximum Losses giving Lines with Each Type of Line Geometry for 4-Conductor Type Line 


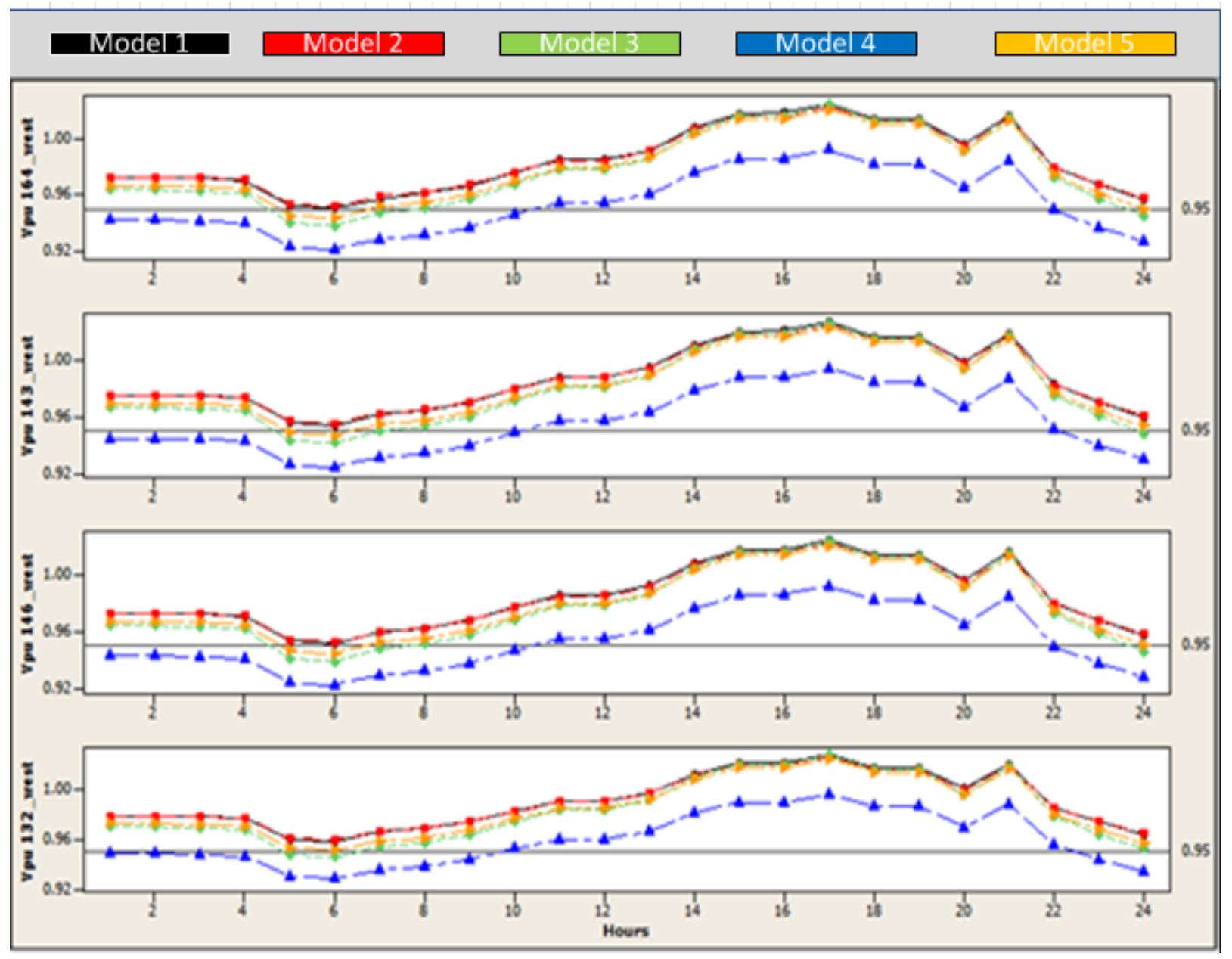

Figure 17 Controller Testing for the Voltage Controllers against Electrical Design Parameter Inconsistencies, Deception Attack and LR Attack

The predictor variables are plotted as shown in figure 17 for a 24 hour period under conventional capacitor control (Model 1), MLR base voltage controller (Model 2), conventional capacitor control with inconsistency in reactance (Model 3), conventional capacitor control with deception attack (Model 4), and conventional capacitor control with LR attack (Model 5) for a 24 hour period with the load and solar generation output curve as given in figure 14. It is observed that voltage in pu calculated from conventional voltage control with inconsistency, deception attack and LR attack have a high deviation from the controller without any abnormality. Moreover, it can be seen that Model 1 and Model 2 maintain the voltage in pu within the permissible range whereas in Model 3, Model 4 and Model 5, the controllers fail to maintain the voltage in pu within the permissible limits for certain hours in a 24 hours period during which the system operations might get disturbed. 


\subsubsection{Cyber Attack Distributed Detection Method}

Unbalanced three-phase distribution power flow solutions are obtained for AEP distribution system under 25 different network conditions. Data sets of voltage in pu for all the buses in the system are calculated using OpenDSS. As discussed in section 3.3 four clusters sets are formed as shown in figure 18 and six agent pairs are identified. These data sets are divided into different clusters using the k-means clustering method done in Matlab. Only four clusters are made as with increase in number of clusters the separation between them decreases and decreasing the number of clusters results in fewer agents. It can be observed from the silhouette plot in the figure 18, that most points in the first, third and fourth cluster have a large silhouette value, greater than 0.8 , indicating that these clusters are somewhat separated from neighboring clusters. However, the second cluster contains many points with low silhouette values with negative values also, indicating that this cluster is not well separated.

Models based on MLR obtained for the agent pairs are defined in table 4. The coefficient of correlation is greater than 0.98 and MSE close to zero show good fit and also the agent pairs belong to different cluster sets. Figure 19 shows the normal probability plot of the residual for the statistical models for attack detection and indicate the goodness of fit of all the models.

The total number of buses, the centroid value and the two elected buses based on the lowest sum of absolute values are shown in table 5 for each cluster. Total six elected pairs are identified from the whole network. Linear regression analysis is done on the elected pairs in Minitab software to get six local agents. 


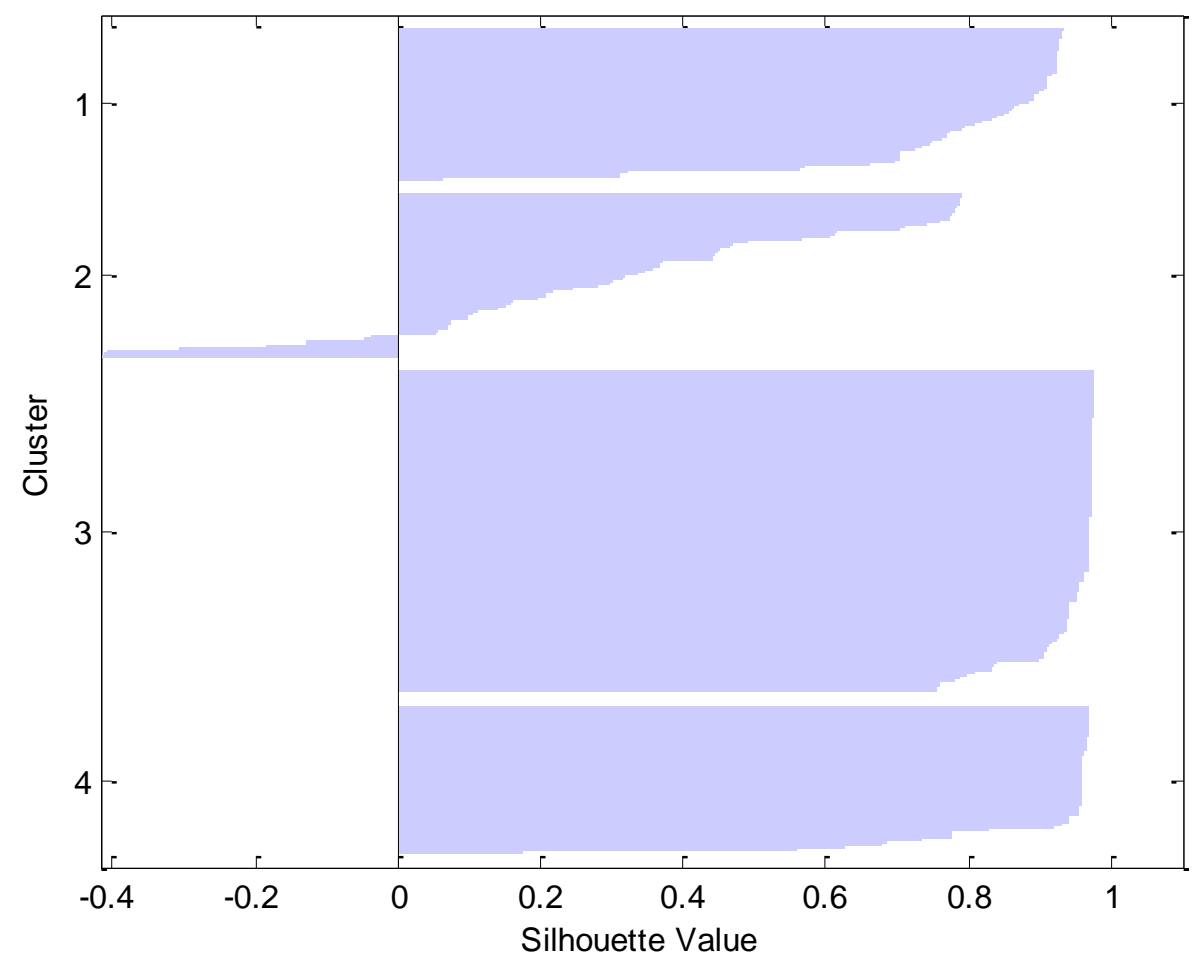

Figure 18 Cluster Division for AEP Feeder 1 Network

Table 4. Statistical Models for Attack Detection.

\begin{tabular}{|c|c|c|c|c|}
\hline Bus m & Model (Bus n) & $\begin{array}{l}\text { Cluster } \\
(\mathbf{m}, \mathbf{n})\end{array}$ & $\mathbf{R}^{2}$ & MSE \\
\hline$\hat{V} p u_{172 \text { west }}$ & $0.519912 * \hat{V} p u_{74 \_w e s t}+0.48766$ & 1,2 & 0.9938 & $1.42 \mathrm{e}-07$ \\
\hline$\hat{V} p u_{172 \text { west }}$ & $1.553568 * \widehat{V} p u_{3_{-} \text {west }}-0.56342$ & 1,3 & 0.9997 & $6.62 \mathrm{e}-09$ \\
\hline$\hat{V} p u_{172 \_w e s t}$ & $0.257543 * \hat{V} p u_{\text {fict } 128 \text { west }}+0.744397$ & 1,4 & 0.9999 & $2.72 \mathrm{e}-10$ \\
\hline$\hat{V} p u_{\text {fict173_west }}$ & $0.530525 * \hat{V} p u_{75_{-} w e s t}+0.476897$ & 1,2 & 0.9935 & $1.54 \mathrm{e}-07$ \\
\hline$\hat{V} p u_{75 \text { west }}$ & $0.497426 * \hat{V} p u_{127 \text { west }}+0.491538$ & 2,4 & 0.9934 & $5.54 \mathrm{e}-07$ \\
\hline$\hat{V} p u_{16 \text { west }}$ & $0.167145 * \hat{V} p u_{127 \text { west }}+0.840426$ & 3,4 & 0.9998 & $1.74 \mathrm{e}-09$ \\
\hline
\end{tabular}


Table 5. Total Number of Buses and the Centroid Value.

\begin{tabular}{lll}
\hline Clusters & $\begin{array}{l}\text { Number of } \\
\text { Buses }\end{array}$ & Centroid Value \\
\hline Cluster 1 & 82 & 0.98885 \\
Cluster 2 & 76 & 0.96344 \\
Cluster 3 & 161 & 0.99913 \\
Cluster 4 & 74 & 0.94312 \\
\hline
\end{tabular}
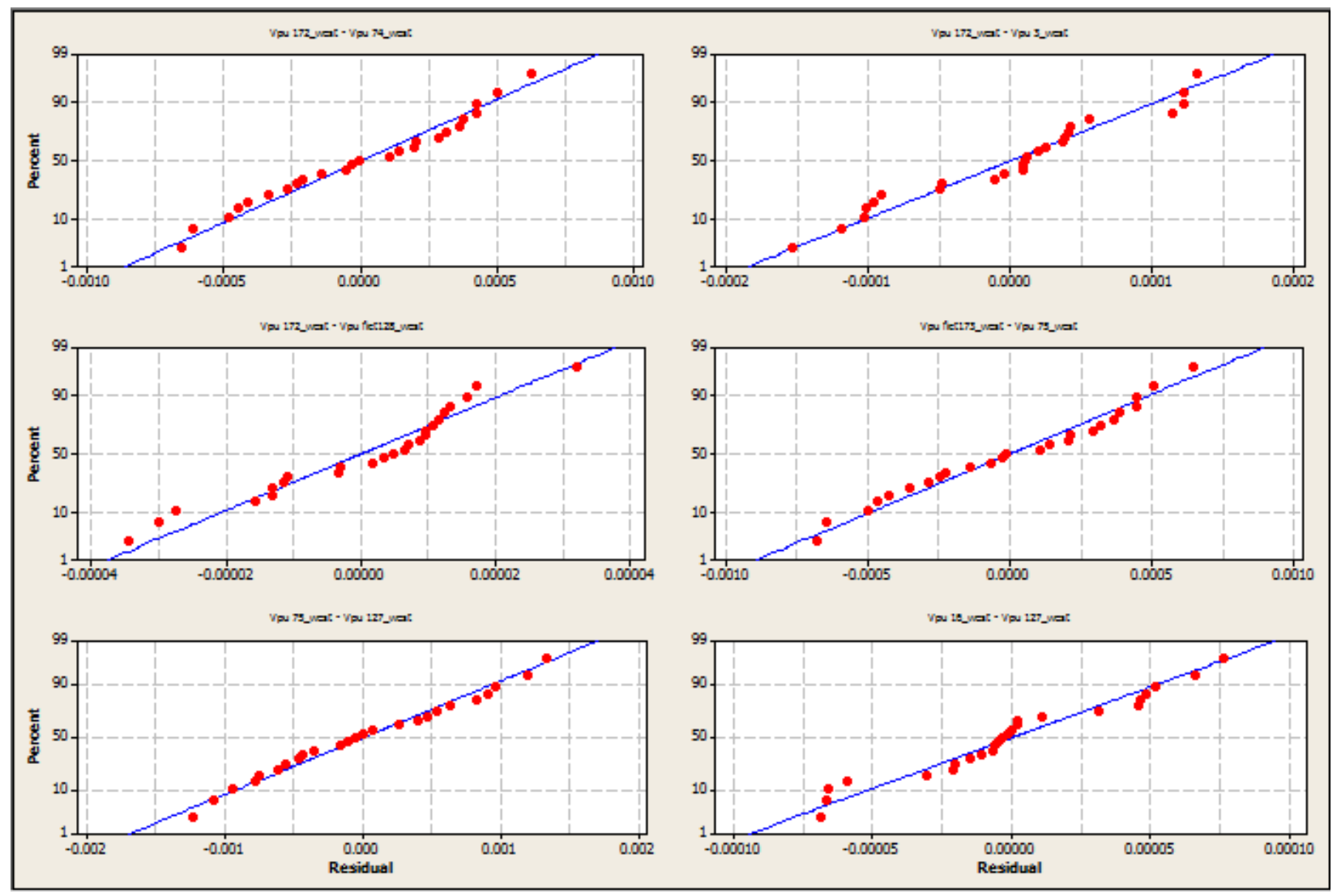

Figure 9 Normal Probability Plots for the Regression Models of the Agents

The cyber-attack distributed detection method is tested against deception attack and LR attack on the AEP Feeder 1 with different attack scenarios constructed in OpenDSS.

1. Deception Attack

Scenario 1: No attack 
Scenario 2: Increase in var injection of capacitor at 131_west from $150 \mathrm{kVar}$ to $300 \mathrm{kVar}$ (50\%)

Scenario 3: Decrease in var injection of capacitor at 131_west from $150 \mathrm{kVar}$ to $75 \mathrm{kVar}(50 \%)$

Scenario 4: Increase in var injection of capacitor at 131_west from $150 \mathrm{kVar}$ to $180 \mathrm{kVar}(20 \%)$

2. Load Redistribution Attack

Scenario 1: No attack

Scenario 2: Increase in load 644_7231501-1 from $75 \mathrm{~kW}$ to150 kW and decrease in load 382_7231503-1 from $222.5 \mathrm{~kW}$ to $147.5 \mathrm{~kW}$

Scenario 3: Increase in load 25_7231504-1 from $160 \mathrm{~kW}$ to $270 \mathrm{~kW}$ and decrease in load $382 \_7231503-1$ from $222.5 \mathrm{~kW}$ to $112.5 \mathrm{~kW}$

Scenario 4: Decrease in load 331_7231503-1 from $115 \mathrm{~kW}$ to $60 \mathrm{~kW}$ and increase in load 322_7231503-1 from $75 \mathrm{~kW}$ to $130 \mathrm{~kW}$

Figure 20 and figure 21 show the local decision by each agent and the final decision for the scenarios presented above for deception attack and load redistribution attack respectively. Here, the green blocks indicate "no cyber-attack" decision whereas red blocks indicate "cyber-attack" decision provided by the agents. It is observed that the proposed detection method provide accurate decision for all the scenarios for both the attacks. It is inferred that for the deception attack case, most of the agents detect the attack as the effect of the change in the var injection by the capacitor causes significant effect on the per unit voltages in the system. Whereas for the LR attack case, comparatively fewer agents detect the attack, as the individual loads that are modified by the attack has insignificant value when compared to the total load of the system in AEP Feeder 1. 


\begin{tabular}{|l|l|l|l|l|l|l|l||}
\hline Attacks & Agent 1 & Agent 2 & Agent 3 & Agent 4 & Agent 5 & Agent 6 & Decision \\
\hline Scenario 1 & & & & & & & \\
\hline Scenario 2 & & & & & & & \\
\hline Scenario 3 & & & & & & & \\
\hline Scenario 4 & & & & & & & \\
\hline
\end{tabular}

Figure 20 Decision Table for Deception Attack

\begin{tabular}{|l|l|l|l|l|l|l||l||}
\hline Attacks & Agent 1 & Agent 2 & Agent 3 & Agent 4 & Agent 5 & Agent 6 & Decision \\
\hline Scenario 1 & & & & & & & \\
\hline Scenario 2 & & & & & & & \\
\hline Scenario 3 & & & & & & & \\
\hline Scenario 4 & & & & & & & \\
\hline
\end{tabular}

Figure 21 Decision Table for LR Attack 


\section{Chapter 6: CONCLUSION AND FUTURE WORK}

\subsection{Conclusion}

In this thesis, a novel multiple linear regression based method to control the bus voltages in a distribution system with renewable sources is proposed. A statistical distributed detection technique based on local decision making agents is proposed and validated. These proposed methods are demonstrated on a real world distribution system feeder, AEP system Feeder 1 modelled in OpenDSS whereas the regression modellings are done in Minitab software. The conclusions drawn from both these works have been presented in this section.

\subsubsection{Voltage Controller Strategy}

In this thesis, a robust and reliable voltage controller based on multiple linear regressions to maintain the voltage profile in a distribution system with distributed generators (DG) connected to it is developed. The proposed controller is validated on IEEE 13 bus distribution system and American Electric Power System feeder modeled in OpenDSS.

The results showed that the developed strategy for voltage control involve exact network simulations initially, but once the models are designed no further calculations are required unless the network topology changes. Also the effectiveness of the proposed method is shown in the presence of inconsistencies in electrical design parameters, data integrity attacks and LR attacks which make them a viable alternative to the conventional capacitor controller.

\subsubsection{Distributed cyber-attack detection technique}

A regression based distributed detection algorithm having local detection agents is developed for detection of cyber-attack in a distribution system with DG connected to it. An algorithm is developed to select a certain number of buses in the system to be declared as elected buses pairs and linear regression based local agents are developed from the elected pairs of buses. The cyber-attacks and detection technique are developed and validated in AEP feeder modeled in OpenDSS. 
The results showed that the developed technique for cyber-attack detection involve exact network simulations initially, but once the methods are designed no further calculations are required unless the network topology changes. The elected buses for developing the agents being distributed sparsely along the feeder and having really high correlation among them detected all the attack scenarios with accurate precision.

\subsection{Future Work}

Voltage control and cyber-attack detection techniques in the present smart grid depend on the real time data communication and validation of the variables affecting them respectively in a distribution system. Also the frequently changing topology of the distribution system should be accurately modeled and in a quick span of time for a better and accurate assessment.

For our study, the variations of the tap settings of the voltage regulators can be included in the regression analysis when forming the statistical models for voltage control and attack detection. Also if there is some provision of updating these models after some specified interval of time or after some change in the topology in the distribution system is detected then these statistical models can be applied to the real world. The number of chosen buses can be increased to increase the total agents in the distribution system for further increasing the accuracy for detection of cyber-attack. 


\section{References}

1. "Smart Grid: An Introduction”, Program Concept paper, U.S. Department of Energy, 2001.

2. Rabinowitz M., "Power Systems of the Future", IEEE Power Engineering Review, 2000.

3. Chen C., Zhu Y., Xu Y., "Distributed generation and Demand Side Management", Proceedings of the 2010 China International Conference on Electricity Distribution, 1316 Sept. 2010, pp. 1-5.

4. Guan F. H., Zhao D. M., Zhang X., Shan B.T., Liu Z., "Research on distributed generation technologies and its impacts on power system", Proceedings of the International Conference on Sustainable Power Generation and Supply, 6-7 Apr. 2009, pp. 1-6.

5. Driesen J., Belmans R., "Distributed generation: challenges and possible solutions", Proceedings of the IEEE Power Engineering Society General Meeting, 2006, pp. 1-8.

6. Chowdhury B. H., Sawab A.W., "Evaluating the value of distributed photovoltaic generations in radial distribution systems" IEEE Transactions on Energy Conversion, vol. 11, no. 3, Sept. 1996.

7. Fitzer J., Dillon W. E., "Impact of Residential Photovoltaic Power Systems on the Distribution Feeder,” IEEE Power Engineering Society Winter Meeting, 1986.

8. McMillan R., "Siemens: Stuxnet worm hit industrial systems," COMPUTERWorld, 14 Sept. 2010.

9. Cherry S., Langner R., "How Stuxnet Is Rewriting the Cyberterrorism Playbook", IEEE Spectrum, 13 Oct. 2010.

10. U.S.-Canada Power System Outage Task Force, "Final Report on the August 14, 2003

Blackout in the United States and Canada: Causes and Recommendations", April 2004. Online: https://reports.energy.gov/BlackoutFinal-Web.pdf.

11. Annual report 2011, The Repository for Industrial Security Incidents (RISI), Online: http://www.securityincidents.net/index.php/products/indepth/risi_annual_report/. 
12. 2011 Report on control system cyber security incidents, online: http://community.controlglobal.com/content/risi-cyber-incident-report-2011-calendaryear-out-risicybersecurity-.

13. Sridhar S., Hahn A., Govindarasu M., "Cyber-Physical System Security for the Electric Power Grid”, Proceedings of the IEEE, vol. 100, no. 1, Jan. 2012, pp. 210-224.

14. Goetz E., Shenoi S., “Critical Infrastructure Protection,” Springer Nov. 2009.

15. Coughlan B.W., Lubkeman D.L., Sutton J., "Improved control of capacitor bank switching to minimize distribution systems losses", Proceedings of the $22^{\text {nd }}$ Annual North American Power Symposium, 15-16 Oct. 1990, pp. 336-345.

16. Bunch J.B., Miller R.D., Wheeler J.E., "Distribution system integrated voltage and reactive power control", IEEE Transactions on Power Apparatus and Systems, vol. PAS101, no. 2, Feb. 1982, pp. 284-289.

17. Miu K.N., Hsiao-Dong C., Darling G., "Capacitor placement, replacement and control in large scale distribution systems by a GA based 2 stage algorithm”, IEEE Transactions on Power Systems, vol. 12, no. 3, Aug. 1997, pp. 1160-1166.

18. Kersting W.H., "Distribution feeder voltage regulation control", IEEE Transactions on Industry Applications, vol. 46, no. 2, Mar.-Apr. 2010, pp. 620-626.

19. Baran M.E., Wu F.F., "Optimal capacitor placement on radial distribution systems", IEEE Transactions on Power Delivery, vol. 4, no. 1, Jan. 1989, pp. 725-734.

20. Borozan V., Baran M.E., Novosel D., "Integrated Volt/var control in distribution systems”, IEEE Power Engineering Society Winter Meeting, 2001, pp. 1485-1490.

21. Mirhoseini S. H., Hosseini S.M., Ghanbari M., Ahamadi M., “A new improved adaptive imperialist competitive algorithm to solve the reconfiguration problem of distribution systems for loss reduction and voltage profile improvement", International Journal of Electrical Power \& Energy Systems, vol. 55, Feb. 2014, pp.128-143.

22. Jin-Cheng W., Hsiao-Dong C., Miu K.N., Darling G., "Capacitor placement and real time control in large scale unbalanced distribution systems: loss reduction formula, problem formulation, solution methodology and mathematical justification”, Proceedings of IEEE Transmission and Distribution Conference, 15-20 Sept. 1996, pp. 236-241. 
23. Jin-Cheng W., Hsiao-Dong C., Miu K.N., Darling G., "Capacitor placement and real time control in large-scale unbalanced distribution systems: numerical studies", IEEE Transactions on Power Delivery, vol. 12, no. 2, Apr. 1997, pp. 959-964.

24. Klienberg M., Miu K.N., "A study of distributed capacitor control of electric power distribution systems", North American Power Symposium, 4-6 Aug.2011, pp. 1-6.

25. Klienberg M., Miu K.N., Segal N., Lehmann H., Figura T.R., "A partitioning method for distributed capacitor control of electric power distribution systems", IEEE Transactions on Power Systems, vol. 29, no. 2, Mar. 2014, pp. 637-644.

26. Shen Z., Wang Z., Baran M.E., "Optimal volt/var control strategy for distribution system with multiple voltage regulating devices", IEEE PES Transmission and Distribution Conference and Exposition, 7-10 May 2012, pp. 1-7.

27. Jen-Hao T., Chia-Yen C., Chi-Fa C., Yi-Hwa L., "Optimal capacitor control for unbalanced distribution systems with distributed generations", IEEE International Conference on Sustainable Energy Technologies, 24-27 Nov. 2008, pp. 755-760.

28. Amin S., Cardenas A., Sastry S., "Safe and secure networked control systems under denial-of-service attacks," in Hybrid Systems: Computation and Control, vol. 5469, Apr. 2009, pp. 31-45.

29. Huangc Y.-L., Cárdenasa A. A., Aminb S., Linc Z.-S., Tsaic H.-Y., Sastrya S., "Understanding the physical and economic consequences of attacks on control systems," International Journal of Critical Infrastructure Protection, vol.2, no. 3, Oct. 2009, pp. 7283.

30. Rahman M.A., Mohsenian-Rad H., "False Data Injection Attacks with Incomplete Information against Smart Power Grids", IEEE Global Communications Conference, 3-7 Dec. 2012, pp. 3153-3158.

31. Hug G., Giampapa J.A., "Vulnerability Assessment of AC State Estimation With Respect to False Data Injection Cyber-Attacks", International Journal of Critical Infrastructure Protection, vol. 2, no. 3, Oct. 2009, pp. 72-83.

32. Liu Y., Ning P., Reiter M.K., "False data injection attacks against state estimation in electric power grids," Proceedings of $16^{\text {th }}$ ACM conference on Computer and communication security, Oct. 2010, pp. 21-32. 
33. Dan G. and Sandberg H., "Stealth attacks and protection schemes for state estimators in power systems," Proceedings of $1^{\text {st }}$ IEEE International Conference Smart Grid Communication, 2010, pp. 214-219.

34. Teixeira A., Amin S., Sandberg H., Johansson K.H., Sastry S.S., “Cyber security analysis of state estimators in electric power systems," Proceedings of $49^{\text {th }}$ IEEE Conference on Decision Control, 2010, pp.5991-5998.

35. Yuan Y., Li Z., Ren K., "Modeling Load Redistribution Attacks in Power Systems," IEEE Transactions on Smart Grid, vol. 2, no. 2, June 2011, pp. 382-390.

36. Liu X. and Li Z., "Local Load Redistribution Attacks in Power Systems With Incomplete Network Information,” IEEE Transactions on Smart Grid, vol. 5, no. 4, July 2014, pp. 1665-1676.

37. Yuan Y., Li Z., Ren K., "Quantitative Analysis of Load Redistribution Attacks in Power Systems," IEEE Transactions on Parallel and Distributed Systems, vol. 23, no. 9, Sept. 2012, pp. 1731-1738.

38. Sou K.C., Sandberg H., Johansson K.H., "Detection and Identification of Data Attacks in Power System,” American Control Conference, 27-29 June 2012, pp. 3651-3656.

39. Bobba R.B., Rogers K.M., Wang Q., Khurana H., Nahrstedt K., Overbye T.J., "Detecting False Data Injection Attacks on DC State Estimation”, $1^{\text {st }}$ Workshop on Secure Control Systems, Apr. 2010.

40. Dorfler F., Pasqualetti F., Bullo F., "Distributed Detection of Cyber-Physical Attacks in Power Networks: A Waveform Relaxation Approach," $49^{\text {th }}$ Annual Allerton Conference on Communication, Control, and Computing, 28-30 Sept. 2011, pp. 1486-1491.

41. Giani A., Bitary E., Garciay M., McQueenz M., Khargonekarx P., Poolla K., "Smart Grid Data Integrity Attacks: Characterizations and Countermeasures,” IEEE International Conference on Smart Grid Communications, 17-20 Oct. 2011, pp. 232-237.

42. Kim T.T., Poor H.V., "Strategic Protection against Data Injection Attacks on Power Grids," IEEE Transactions on Smart Grid, vol. 2, no. 2, June 2011, pp. 326-333.

43. Sridhar S., Manimaran G., "Data integrity attacks and their impacts on SCADA control system,” IEEE Power and Energy Society General Meeting, July 2010, pp. 1 -6.

44. Sridhar S., Manimaran G., "Data integrity attack and its impacts on voltage control loop in power grid," IEEE Power and Energy Society General Meeting, July 2011, pp. 1 -6. 
45. OpenDSS Manual, Electric Power Research Institute, Jul 2010. Available: http://sourceforge.net/projects/electricdss

46. http://www.smartgrid.epri.com/doc/OpenDSS Level 1 Training.pdf

47. Ramachandran V., Solanki J., Solanki S.K., "Steady state analysis of high penetration PV on utility distribution feeder," IEEE PES Transmission and Distribution Conference and Exposition, May 2012, pp. 1 -6.

48. Yan X., Su X. G., "Linear Regression Analysis: Theory and Computing”. 
West Virginia University Electronic

Problem/Project/Research Report

Signature Form

Student Name:

JOSHI

VIVEK

Student ID \#: $\quad 800012518$ (First)

(Middle)

Degree:

Non-WVU Email Account: vivekjos Li 1858@gmail.co.

Document Type:

Master's

Thesis Problem/Project/Research Report

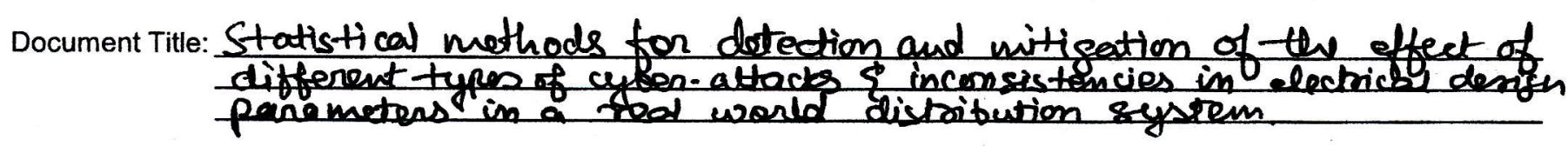

\section{Student Agreement:}

I hereby certify that, if appropriate, I have obtained and attached hereto a written permission statement from the owners of each third party copyrighted matter to be included in my thesis, dissertation, project report, or other research material, allowing distribution as specified upon deposit.

I hereby grant to West Virginia University and its agents the non-exclusive license to archive and make accessible, under the conditions selected upon deposit, my above mentioned document in whole or in part in all forms of media, now or hereafter known. I retain ownership rights as specified in the WVU copyright policy to the copyright of the abovementioned document. I also retain the right to use in future works (such as articles or books) all or part of this abovementioned document.

\section{Review and Acceptance:}

The above mentioned document has been reviewed and accepted by the student's advisory committee. The undersigned agree to abide by the statements above, and agree that this Signature Form updates any and all previous Signature Forms submitted heretofore.

Signed:

Committee:

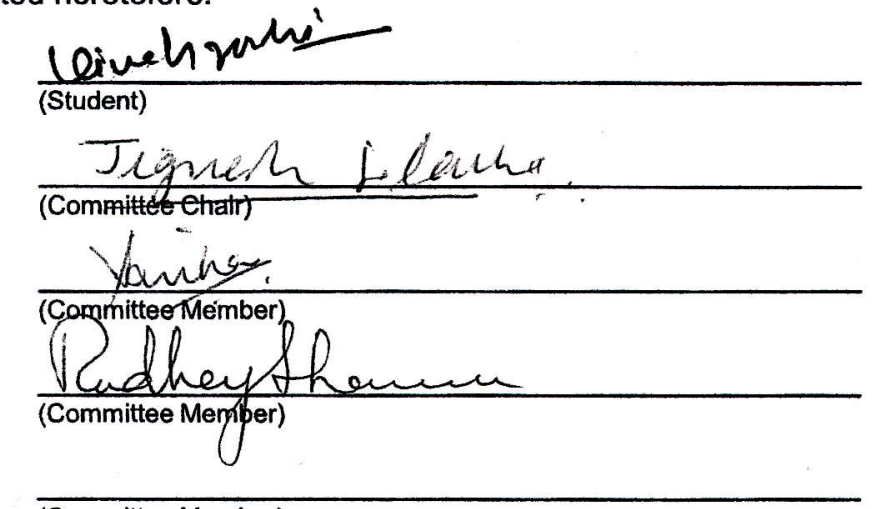

(Committee Member)
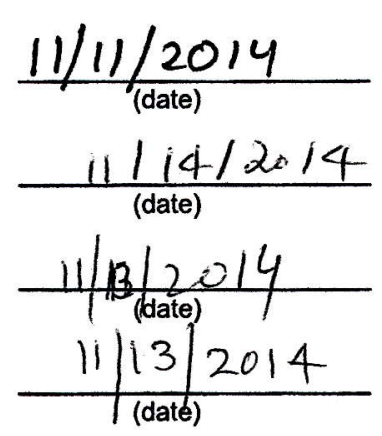

(date) 
APPROVAL OF EXAMINING COMMITTEE

Jigued Lolauht.

Dr. Jignesh Solanki, Ph.D

Committee Chairman

Sarine

Dr. Sarika Khushalani Solanki, Ph.D

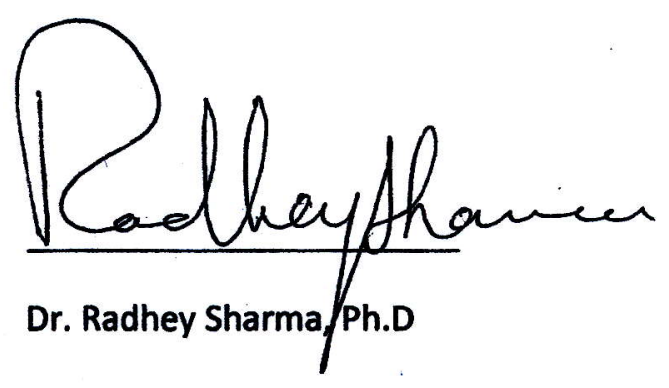

